\title{
Connectomes across development reveal principles of brain maturation in C. elegans
}

\author{
Daniel Witvliet ${ }^{1,2}$, Ben Mulcahy ${ }^{1, a}$, James K. Mitchell ${ }^{3,4, a}$, Yaron Meirovitch ${ }^{4,5}$, Daniel R. Berger ${ }^{4}$, Yuelong Wu ${ }^{4}$, Yufang Liu ${ }^{1}$, Wan \\ Xian Koh ${ }^{1}$, Rajeev Parvathala ${ }^{5}$, Douglas Holmyard ${ }^{1}$, Richard L. Schalek ${ }^{4}$, Nir Shavit ${ }^{5}$, Andrew D. Chisholm ${ }^{6}$, Jeff W. Lichtman $^{4,7, *}$, \\ Aravinthan D.T. Samuel ${ }^{3,4, *}$, and Mei Zhen ${ }^{1,2,8, *}$

\begin{abstract}
${ }^{1}$ Lunenfeld-Tanenbaum Research Institute, Mount Sinai Hospital, Toronto, ON, Canada, ${ }^{2}$ Department of Molecular Genetics, University of Toronto, Toronto, ON, Canada, ${ }^{3}$ Department of Physics, Harvard University, Cambridge, MA, ${ }^{4}$ Center for Brain Science, Harvard University, Cambridge, MA, ${ }^{5}$ Computer Science and Artificial Intelligence ${ }^{7}$ Department of Molecular and Cellular Biology, Harvard University, Cambridge, MA, ${ }^{8}$ Department of Physiology, University of Toronto, Toronto, ON, Canada, ${ }^{\text {a These authors }}$ contributed equally to this work.
\end{abstract} \\ Laboratory, Massachusetts Institute of Technology, MA, ${ }^{6}$ Division of Biological Sciences, Section of Cell and Developmental Biology, University of California, San Diego, CA,
}

*For correspondence: jeff@mcb.harvard.edu (JWL); samuel@physics.harvard.edu (ADTS); zhen@lunenfeld.ca (MZ).

\begin{abstract}
From birth to adulthood, an animal's nervous system changes as its body grows and its behaviours mature. However, the extent of circuit remodelling across the connectome is poorly understood. Here, we used serial-section electron microscopy to reconstruct the brain of eight isogenic $C$. elegans individuals at different ages to learn how an entire wiring diagram changes with maturation. We found that the overall geometry of the nervous system is preserved from birth to adulthood, establishing a constant scaffold upon which synaptic change is built. We observed substantial connectivity differences among individuals that make each brain partly unique. We also observed developmental connectivity changes that are consistent between animals but different among neurons, altering the strengths of existing connections and creating additional connections. Collective synaptic changes alter information processing of the brain. Across maturation, the decision-making circuitry is maintained whereas sensory and motor pathways are substantially remodelled, and the brain becomes progressively more modular and feedforward. These synaptic changes reveal principles that underlie brain maturation.
\end{abstract}

\section{Introduction}

The developing nervous system faces multiple challenges. Amid an animal's changing anatomy and fluctuating environment, some circuits must maintain robust outputs, such as locomotion ${ }^{1-4}$. New circuits need to be constructed in order to support new functions, such as reproduction ${ }^{5-7}$. Moreover, to adapt and learn, the nervous system must make appropriate changes in existing circuits upon exposure to internal and external cues ${ }^{8}$. The nervous system employs a variety of adaptive mechanisms to overcome these challenges. In the Drosophila nerve cord, synaptic density of mechanosensory neurons scales to body size from first to third instar larvae ${ }^{4}$. In the spinal cord of the zebrafish larva, descending neurons lay down tracks chronologically, coinciding with the maturation of swimming behaviours ${ }^{7}$. In the mouse visual circuit, postnatal synaptic remodelling is shaped by intrinsic activity as well as visual stimuli ${ }^{9}$. The prevalence of anatomical changes in the nervous system must accommodate both growth and experience.

Anatomical changes occur at many levels, from individual synapses to global organization of brain networks ${ }^{10}$. An assortment of genetic and cellular factors have been found to affect morphological and functional maturation of synapses ${ }^{11,12}$. Synaptic changes are also likely to be coordinated across developing circuits, giving rise to system-level modifications.
However, developmental principles that describe the synaptic changes that shape the adult brain are unknown.

Interrogating whole-brain maturation at synapse resolution is difficult. High-resolution reconstruction is needed to capture structural changes at individual synapses ${ }^{13}$. These methods must be applied to entire brains, and to brains at different developmental timepoints. Moreover, multiple animals need to be analyzed to assess structural and behavioural heterogeneity. Serial-section electron microscopy (EM) allows reconstruction of neural circuits with synapse resolution ${ }^{14-20}$, but low throughput makes it difficult to compare whole brain samples and comprehensively assess plasticity. EM has been applied to assess wiring differences between species ${ }^{21}$, sexes ${ }^{22}$, genotypes ${ }^{23}$, and ages ${ }^{4,24}$. But previous studies mapped partial circuits or few samples.

The original C. elegans connectome was compiled from the EM reconstruction of partially overlapping regions of at least four adults and an L4 larva ${ }^{25,26}$. A revisit of the $C$. elegans connectome expanded this wiring diagram by re-annotation of original EM micrographs and filled remaining gaps by interpolation ${ }^{22}$. Such compilations make it difficult to assess plasticity or variability between individuals.

Here, we leveraged advances in automation and throughput of EM reconstruction to study of the brain of $C$. elegans - its circumpharyngeal nerve ring and ventral ganglion - across development. We have fully reconstructed the brain of eight isogenic hermaphroditic individuals at different ages from birth to adulthood. These reconstructions provide quantitative assessments for the length, shape, and position of every neural and muscle fibre in the nerve ring, as well as of every physical contact and chemical synapse between neurons, glia, and muscles. Our quantitative comparisons of these developmental connectomes have revealed several organizing principles by which synaptic changes shape the mind of the developing worm.

\section{Results}

\section{EM reconstruction of eight $C$. elegans brains from birth to adulthood}

We leveraged advances in ultra-structural preservation, serial ultra-thin sectioning, and semi-automated imaging ${ }^{27-29}$ to reconstruct the connectivity and morphology of all cells in eight individual isogenic hermaphroditic brains of C. elegans (N2) at various post-embryonic stages (Fig. 1a, Fig. S1, Video 1-2, see Methods). The brain, consisting of the nerve ring and ventral ganglion, includes 162 of the total 218 neurons at birth (L1), 
bioRxiv preprint doi: https://doi.org/10.1101/2020.04.30.066209; this version posted May 22, 2020. The copyright holder for this preprint (which was not certified by peer review) is the author/funder, who has granted bioRxiv a license to display the preprint in perpetuity. It is made available under aCC-BY-NC-ND 4.0 International license.
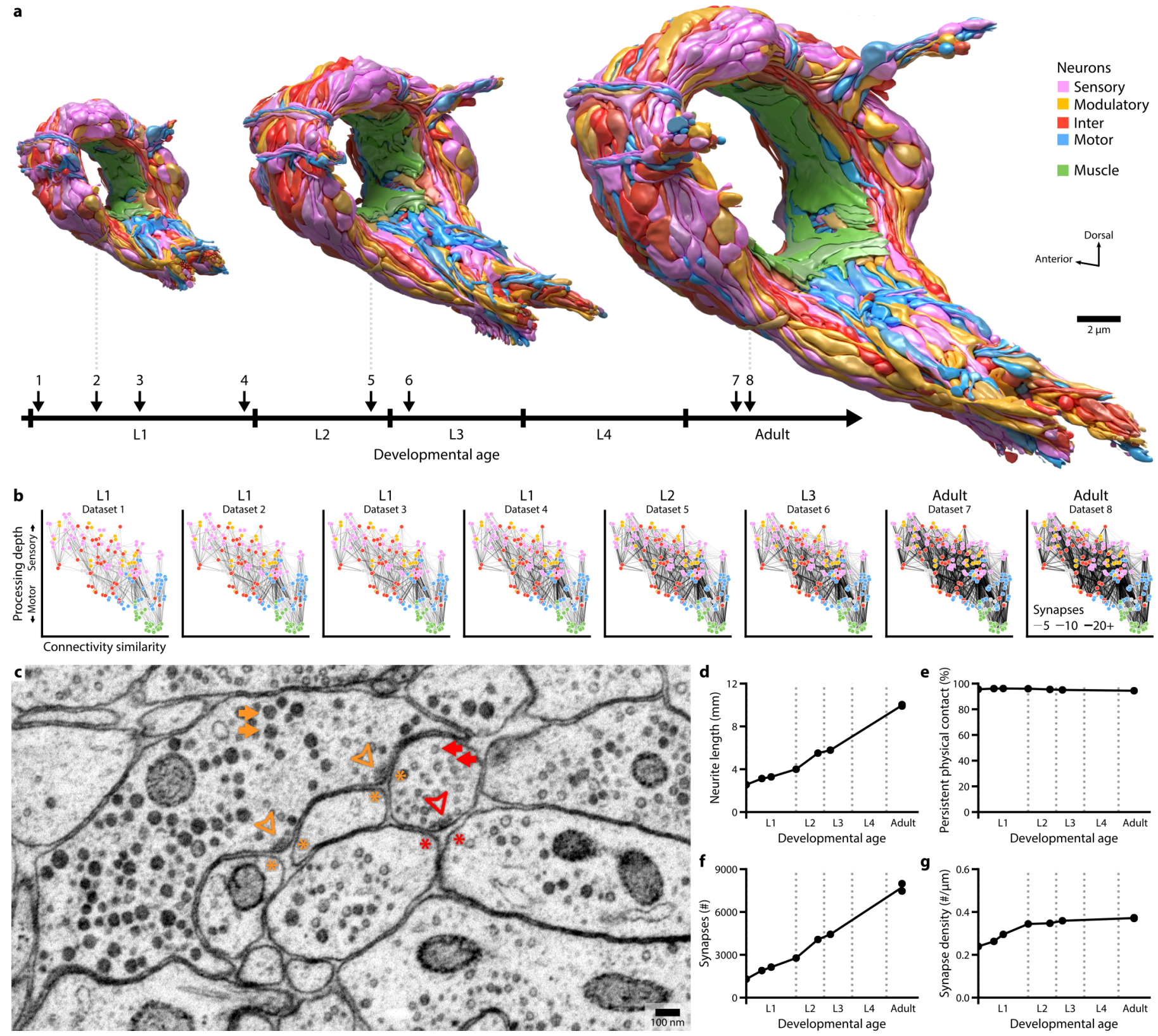

Figure 1. The developing brain maintains its overall geometry. a. Developmental timeline of eight reconstructed brains, with volumetric models shown at three stages. Models include all neurites contained in the neuropil, coloured by cell types. b. Wiring diagrams for all datasets. Each circle represents a cell. Each line represents a connection with at least one chemical synapse between two cells. The line width indicates synapse number. The vertical axis denotes signal flow from sensory perception (top) to motor actuation (bottom); the horizontal axis denotes connectivity similarity (normalized Laplacian eigenvector 2 ) where neurons that share partners are positioned nearby each other ${ }^{26}$. Signal flow and connectivity similarity are based on the accumulated connections from all datasets. c. A representative EM micrograph of the neuropil (from dataset 3 ). Presynaptic termini of classical chemical synapses are characterized by a pool of clear synaptic vesicles (red arrows) surrounding an active zone (red arrowhead). Presynaptic termini of chemical synapses of modulatory neurons are characterized by mostly dense core vesicles (orange arrows) distant from the active zone (orange arrowhead). Postsynaptic cells are marked by asterisks. d. Summed length of all neurites in the brain exhibits linear increase from birth to adulthood. Each data point represents the total neurite length from one dataset. e. Physical contact between neurite pairs at birth that persist across maturation (persistent physical contact) accounts for nearly all of the contact area at every developmental stage. f. Total synapse numbers in the brain exhibits a 6 -fold increase from birth to adulthood. g. Synapse density, the total number of synapses divided by the total neurite length, is maintained after an initial increase.

and 180 of the total 300 neurons in adulthood (Table S1; excluding CANL/R throughout development and HSNL/R until adulthood) ${ }^{25,30}$. It also contains 10 glia and synaptic sites of 32 muscles at all stages. We identified every cell across different EM volumes based on their unique neurite morphology and position $^{25}$. Each neuron was classified as either being sensory, inter, motor, or modulatory (Table S1, Video 2, see Methods).

In each EM volume, every neuron, glia, and muscle were volumetrically segmented and annotated for chemical synapses to generate a connectome of the complete brain (Fig. 1b, Fig. S2, Video 2, see Methods). These reconstructions include classi- cal synapses with mostly clear vesicles and synapses of modulatory neurons with mostly dense core vesicles (Fig. 1c, see Methods). We plotted the wiring diagrams conforming to the direction of information flow from sensory perception (Fig. 1b top layer) to motor actuation (Fig. 1b bottom layer). All connectomes are hosted on an interactive web-based platform at http://nemanode.org/. These datasets allowed for examination of synaptic connectivity in the context of brain geometry, including the shape and size of each neuron as well as the proximity and contact between each neurite. 

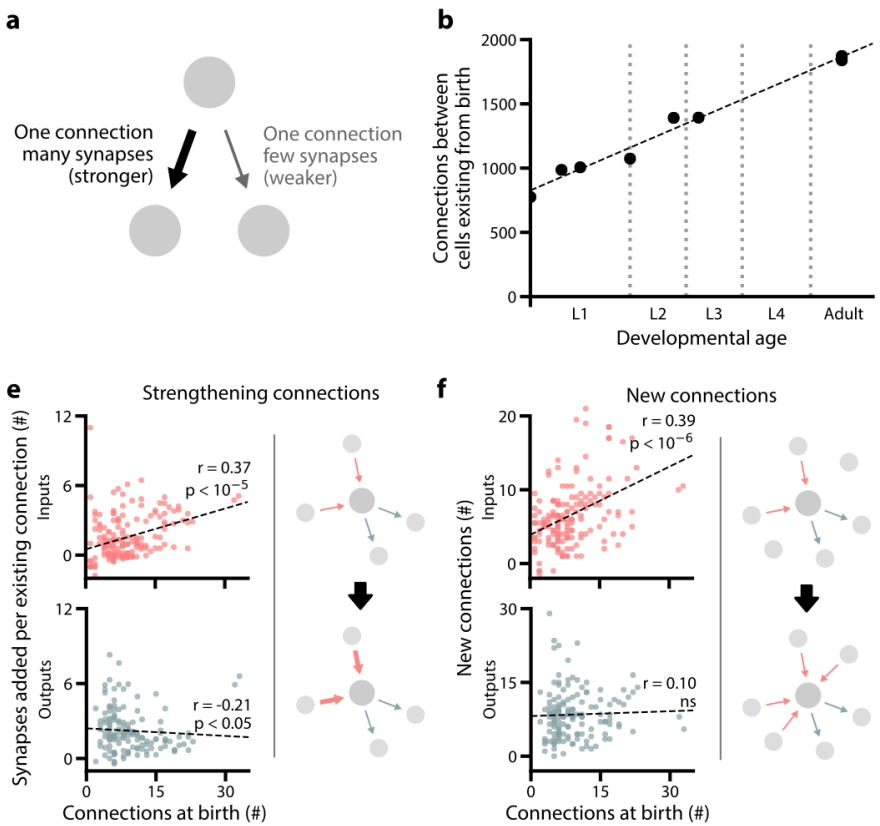

f

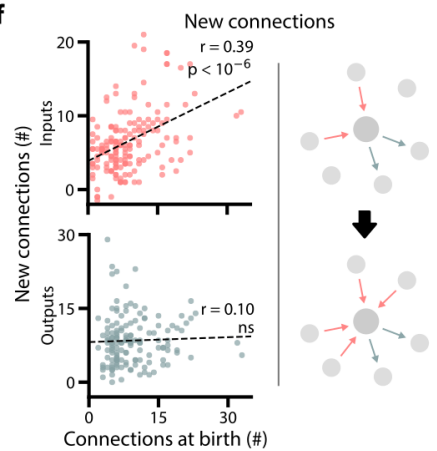

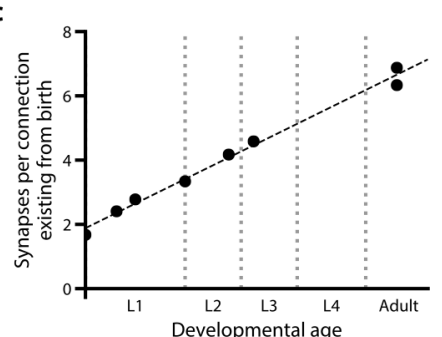

g

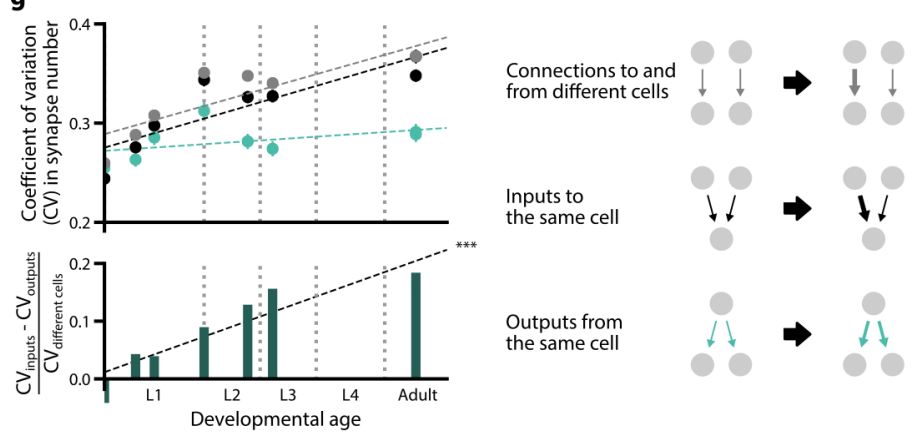

Figure 2. Non-uniform synapse addition reshapes the connectome a. Schematic of two connections. Each connection consists of at least one synapse between two cells. b. The total number of connections in the brain between neurons existing from birth exhibits a 2.4-fold increase. c. The mean number of synapses per connection existing from birth exhibits a 3.9-fold increase. d. The probability of forming a new connection at physical contacts existing from birth. This probability increases with the total contact area between two cells at birth. A new connection is here defined as a connection that appears in adults (datasets 7 and 8 ) but is absent in early L1 stages (datasets 1 and 2 ). ${ }^{\star * \star}$ $r=0.85, p=8.1 \times 10^{-4}$, Spearman's rank correlation. e. Top: neurons with higher number of connections at birth (dataset 1 ) are more likely to receive new synapses at existing input connections by adulthood (averaging datasets 7 and 8). Bottom: no positive correlation is observed at existing output connections. Each data point represents one cell. Significance is calculated using Spearman's rank correlation (top: $p=1.3 \times 10^{-6}, n=165$; bottom: $p=1.1 \times 10^{-2}, n=141$ ). $f$. Top: neurons with higher number of connections at birth (dataset 1) are more likely to establish new input connections by adulthood (averaging datasets 7 and 8 ). Bottom: no correlation is observed at new output connections (bottom). Each data point represents one cell. Significance is calculated using Spearman's rank correlation (top: $p=2.0 \times 10^{-7}, n=165 ;$ bottom: $p=1.2 \times 10^{-1}$, $n=141$ ). g. Top: each data point represents the mean coefficient of variation $(\mathrm{CV})$ in the number of synapses for different sets of connections. The CV of output connections from the same cell is maintained. The CV of input connections to the same cell increases over time, at the same rate as connections to and from different cells. Error bars indicate SE. Bottom: the difference between the mean CV for output and input connections relative to connections between different cells grows over time. ${ }^{\star \star \star} p=6.5 \times 10^{-5}, r=0.97$, Spearman's rank correlation.

\section{Uniform neurite growth maintains brain geometry}

Our volumetric reconstructions revealed striking similarities of brain geometry between developmental stages. The shape and relative position of every neurite in the brain was largely established by birth (Fig. S3a). From birth to adulthood, the total length of neurites underwent a 5-fold increase (Fig. 1d), in proportion to the 5 -fold increase in body length $(\sim 250 \mu \mathrm{m}$ to $\sim 1150 \mu \mathrm{m}$ ). Neurites grew proportionally (Fig. S3b, Video 3), maintaining most physical contact between cells at birth across maturation (Fig. 1e). Only three neuron classes had changes to their primary branching patterns, each growing a new major branch after birth (Fig. S4). Thus, the brain grows uniformly in size without substantially changing the shape or relative position of neurites, maintaining its overall geometry.

In parallel to neurite growth, addition of synapses was extensive. From birth to adulthood, the total number of chemical synapses increased 6-fold, from $\sim 1300$ at birth to $~ 8000$ in adults (Fig. 1f). We found no evidence for systematic synapse elimination. Presynaptic terminals appear as en passant boutons, most often apposing the main neurite of a postsynaptic cell. Small spine-like protrusions ${ }^{25,31}$ were postsynaptic at only $\sim 17 \%$ of synapses in the adult connectome (Fig. S3c). From birth to adulthood, the number of spine-like protrusions increased 5-fold (Fig. S3d), and the proportion of spine-like protrusions apposing presynaptic terminals increased 2-fold (Fig. S3e).

Synapse number increased in proportion to neurite length, maintaining a stable synapse density across most developmental stages. The exception was during the L1 stage, when the increase of total synapse number slightly outpaced that of neurite length, leading to increased synapse density (Fig. 1g). This increase coincided with an increasing left-right symmetry in connectivity (Fig. S3f, S3g). In the adult brain, $~ 90 \%$ of neurons exist as left-right pairs that mirror one another in position, morphology, as well as connectivity. However, some of these neurons exhibited asymmetry in left-right connectivity at birth (Fig. S3f, S3g). The simplest interpretation of this early asymmetry is incompleteness: C. elegans hatches before its brain connectivity has been made symmetric, a process which continues by synapse addition during the first larval stage.

\section{Non-uniform synapse addition reshapes the connec- tome}

From birth to adulthood, addition of synapses both creates new connections and strengthens existing connections. Here, a connection is defined as a pair of cells connected by one or more chemical synapses (Fig. 2a). The 204 cells of the brain were interconnected by $\sim 1300$ total synapses distributed among $\sim 800$ connections at birth (Fig. 2b). Over maturation, addition of synapses strengthened nearly all existing connections. Approximately 4500 synapses were added to connections that were present at birth, such that the mean synapse number per connection increased 4.6-fold, from 1.7 synapses per connection at birth to 6.9 by adulthood (Fig. 2c). In addition, many new connections formed. Approximately 1200 synapses formed between previously non-connected neurons resulting in a 2.4 -fold increase in total number of connections between cells present at birth (Fig. 2b).

Synapse addition did not occur uniformly across the brain. 

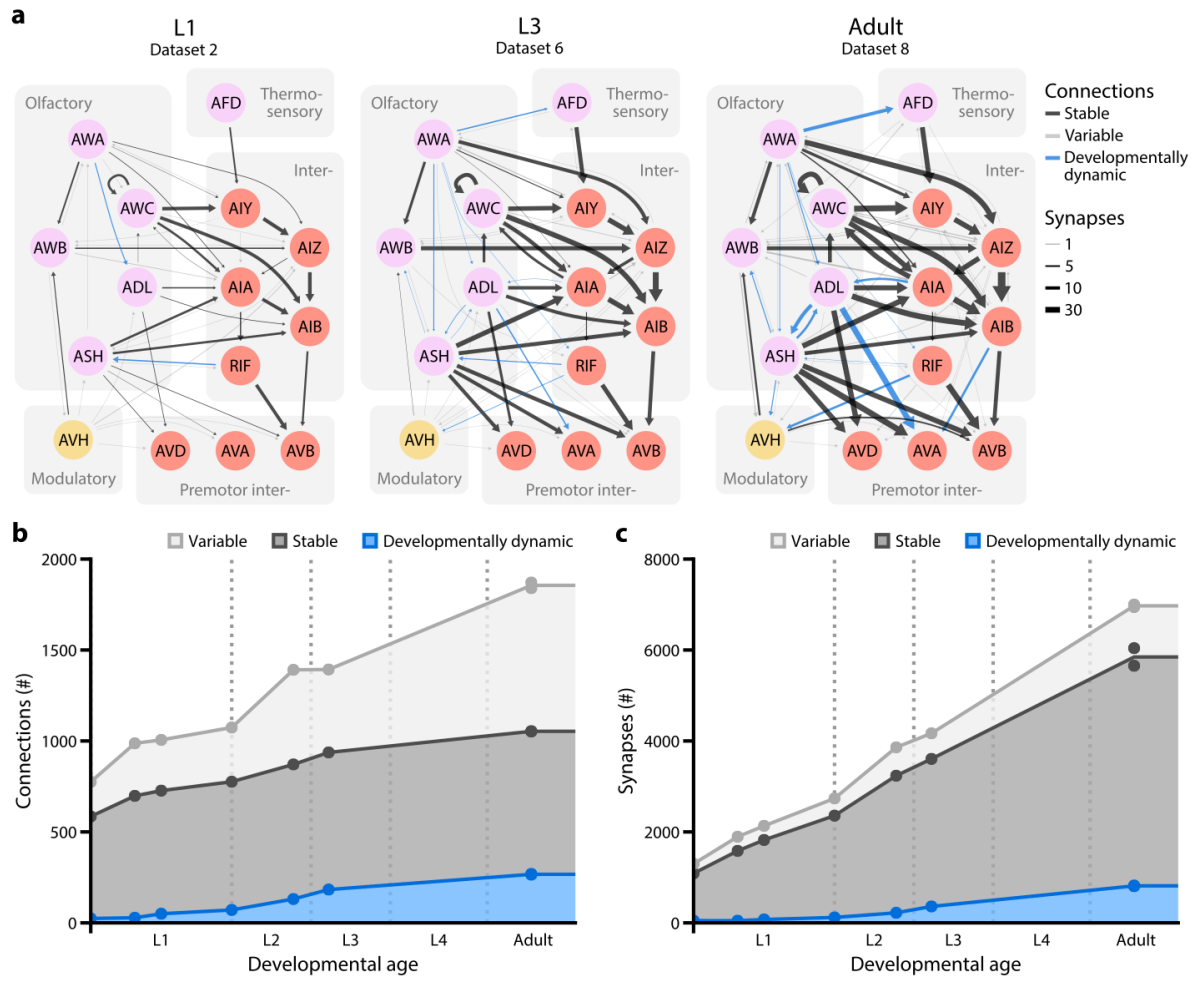

Figure 3. Isogenic individuals have both stereotyped and prevalent variable connections. a. A sensory circuit across maturation. Left: L1 (dataset 2), center: L3 (dataset 6), right: adult (dataset 8 ). Circles represent cells, colour-coded by cell types. Colour-coded lines represent stable (black), developmentally dynamic (blue), and variable (grey) connections. Line width represents synapse number. b. The total number of stable, developmentally dynamic, and variable connections in each dataset. c. The total number of synapses that constitute stable, developmentally dynamic and variable connections in each dataset.

We found preferential synapse addition in multiple contexts.

First, new connections were more likely to form at existing physical contacts between neurons with large contact areas (Fig. 2d). Physical contacts formed at birth appear to create a constant scaffold within which network formation unfolds.

Second, synapse addition was also not uniform between neurons. At birth, it was already evident that some neurons had far more connections than others (Fig. S5a). Neurons with more connections at birth disproportionately strengthened their existing connections over time (Fig. 2e). Interestingly, this disproportionate strengthening only occurred at input connections (Fig. 2e). Neurons with more connections at birth also disproportionately added new input connections in comparison to output connections (Fig. 2f). Thus, during maturation the flow of information is focused onto the most highly-connected neurons at birth.

Lastly, we found that synapse addition to existing connections also changes the relative strengths of a neuron's inputs but not its outputs (Fig. 2g). There was no correlation in the strengthening of existing input connections to each cell from different presynaptic partners (Fig. S5b), leading to a divergence in their relative strengths (Fig. 2g). However, strengthening of the existing output connections from each cell were correlated (Fig. S5b), maintaining their relative strengths (Fig. 2g). Thus, each cell regulates the strengthening of its own synaptic outputs but does not dictate the relative strengthening of its inputs.

\section{Isogenic individuals have both stereotyped and preva- lent variable connections}

We mapped the change in synapse number for each connection across developmental stages. We applied this to classify each connection as either stable, developmentally dynamic, or vari- able (Fig. 3a, Fig. S6). Stable connections were present from birth to adulthood and maintained their relative strength in proportion to one another. Developmentally dynamic connections significantly increased or decreased their relative strength in a stereotyped manner, sometimes forming new connections or eliminating existing connections at specific life stages. Variable connections exhibited no consistent trend in their changing synapse numbers, and were not present in every animal.

In the adult connectome, stable and variable connections each represented $\sim 43 \%$ of the total number of connections, whereas developmentally dynamic connections represented $\sim 14 \%$ (Fig. 3b). Stable connections contained more synapses than variable ones $(6.6 \pm 5.8$ versus $1.4 \pm 1.0$ synapses per connection, respectively, in adult), and thus constituted a large proportion $(\sim 72 \%)$ of total synapses (Fig. 3b). Nonetheless, variable connections were surprisingly common. The number of variable connections in the adult ( $\sim 800)$ is similar to the number of stable connections $(\sim 800)$. The total number of synapses that constitute variable connections in the adult $(\sim 1100)$ is even greater than that of developmentally dynamic connections $(\sim 800)$. Not all variable connections were weak (Fig. S7a). When connections between cell pairs with less than 4 synapses were excluded, variable connections still constituted $\sim 12 \%$ of all connections (Fig. S7b).

We considered the possibility that variability is more prominent during development than in the mature connectome. Hence, a more conservative measure of variability in the adult stage can be made by simply comparing our two adult datasets as well as the original adult connectome ${ }^{25}$. When using these adult datasets to define variability, variable connections still made up $~ 50 \%$ of all connections (Fig. S7c, S7d). Thus, variable connections are prominent in the C. elegans connectome. 


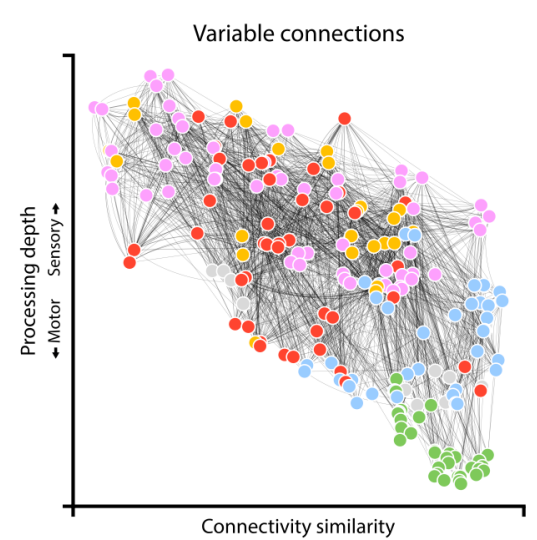

b

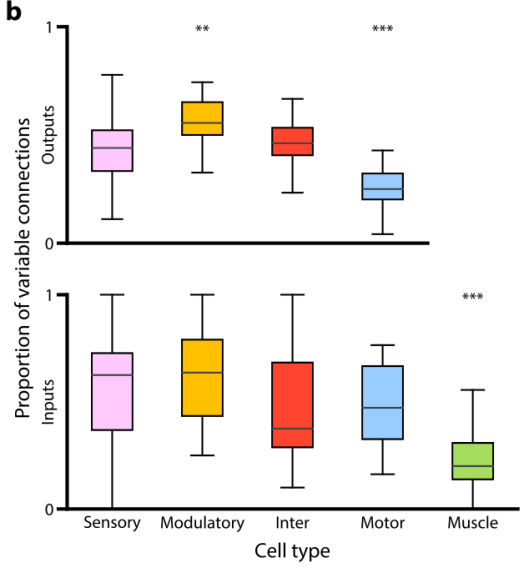

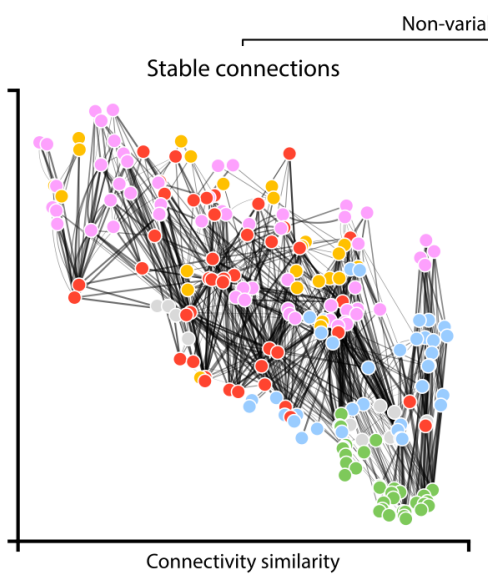

C

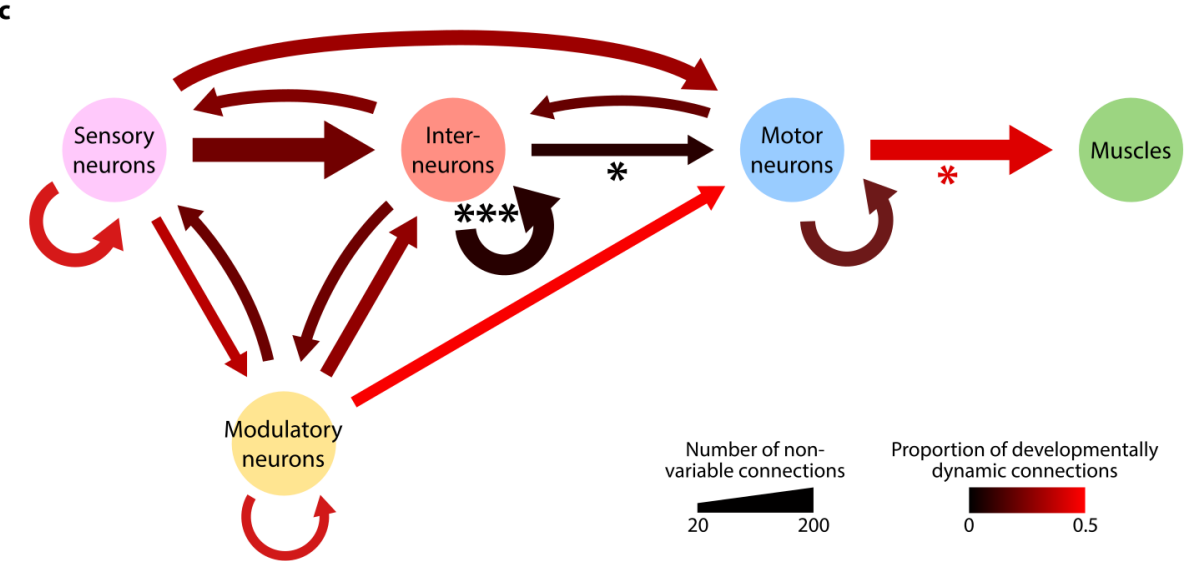

Figure 4. Non-uniform distribution of variable and developmentally dynamic connections. a. Wiring diagrams for variable, stable, and developmentally dynamic connections. Each line represents a connection observed in at least one dataset. Line width indicates the largest number of synapses observed for a connection across datasets. Each circle represents a cell. Cell coordinates are represented as in Fig. 1b. b. Comparison of the proportion of variable and non-variable connections for each cell type. Non-variable connections include stable and developmentally changing connections. Cell types with significantly higher or lower proportions of variable connections are indicated, ${ }^{\star \star} \mathrm{p}<10^{-2}$, ${ }^{* * *} \mathrm{p}<10^{-3}, \mathrm{n}=24-57$, Mann-Whitney $U$ test, FDR adjusted using Benjamini-Hochberg correction. Center line, median; box limits, upper and lower quartiles; whiskers, 1.5x interquartile range; outliers not shown. c. Wiring diagram showing non-variable connections between different cell types. Line width indicates the number of connections. Line color indicates the proportion of developmentally dynamic connections. Lines with significantly different proportions of developmentally dynamic connections are indicated, ${ }^{*} \mathrm{p}<$ $3.7 \times 10^{-2},{ }^{* * *} p=9.5 \times 10^{-6}$, two-tailed Z-test, FDR adjusted using Benjamini-Hochberg correction $\left(n_{\text {inter-inter }}=148, n_{\text {inter-motor }}=52, n_{\text {motor-muscle }}=145\right)$.

\section{Variable connections are not uniformly distributed among cell types}

To visualize the distribution of different classes of connections, we separately plotted their occurrences in the wiring diagram (Fig. 4a). Stable and developmentally dynamic connections represent the portion of the connectome that is shared across animals. Variable connections represent the portion that is unique to each animal.

We quantified the proportion of variable connections in the inputs and outputs of each cell type (Fig. 4b). Modulatory neurons had significantly higher amounts of variability in their output connections than other cell types, whereas motor neurons had significantly less (Fig. 4b upper panel). Consistent with the lowest variability in motor neuron output, muscles exhibited the lowest variability in their inputs (Fig. 4b lower panel).

The higher prevalence of variable connections between certain cell types remained evident when variable connections were defined only between adult datasets (Fig. S8a) and when weak connections were excluded (Fig. S7b). The low variability of connections from motor neurons to muscles could not be simply explained by saturation of their physical contacts by synapses (Fig. S8b). We also considered that neurons with more synapses may exhibit more stochastic synapses or have more annotation errors. However, the proportion of variable connections did not scale with the number of synapses (Fig. S8c-S8f).

Rather, the likelihood of a neuron to generate variable connectivity is likely a property of its cell type. The high stereotypy of synapses from motor neurons to muscles may reflect a requirement for high fidelity in circuits for motor execution. Modulatory neurons, which may secrete monoamines and neuropeptides by volume-release, may have the weakest requirement for precise spatial positions of synaptic outputs because they exert long-range effects.

\section{Interneuron connections are stable during maturation}

Excluding variable connections allows us to properly assess developmental connectivity changes. We found that developmentally dynamic connections were not uniformly distributed among cell types or circuit layers (Fig. 4c). Connections between interneurons, and from interneurons to motor neurons had disproportionately more stable connections than developmentally dynamic connections (Fig. 4c). All other connections, between and from sensory, modulatory, or motor neurons, had many developmentally dynamic connections. Developmentally dynamic connections were particularly prevalent from motor neurons to muscles. Each motor neuron progressively recruited more muscles in a stereotypic pattern (Fig. S6). The abundant but high stereotypy of this developmental connectivity change means that motor neurons exhibit the lowest proportion of vari- 


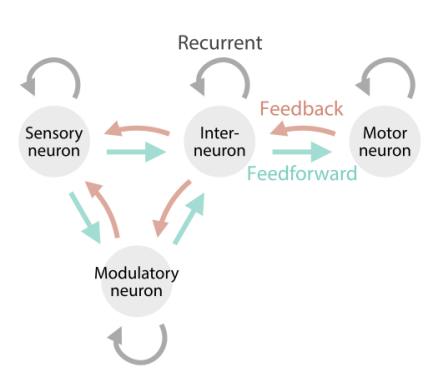

b

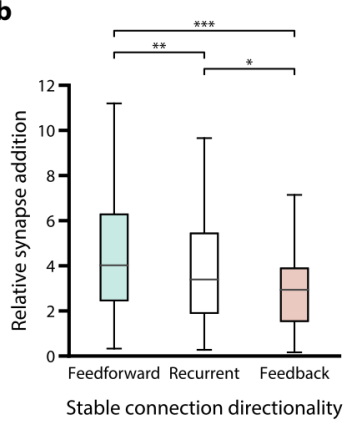

C

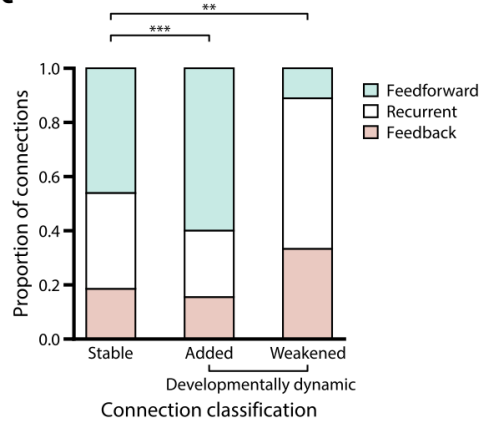

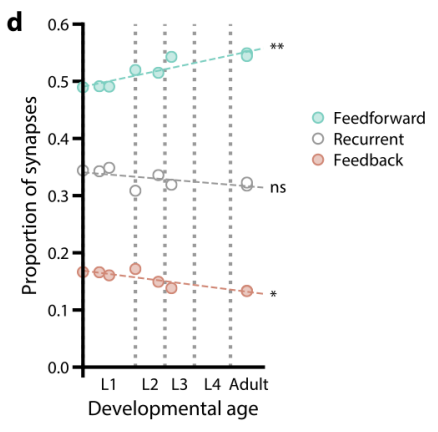

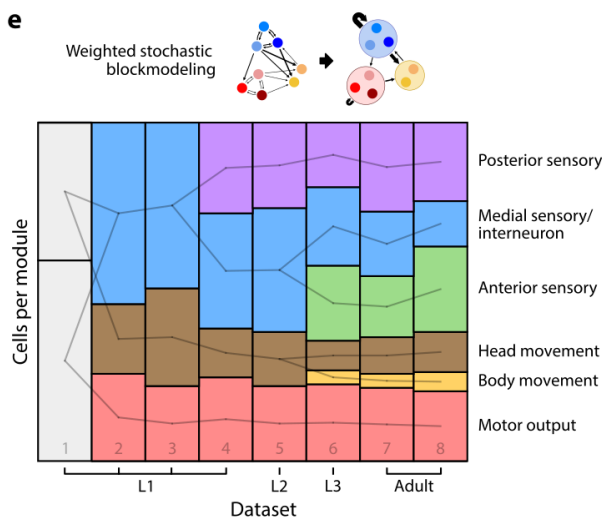

f

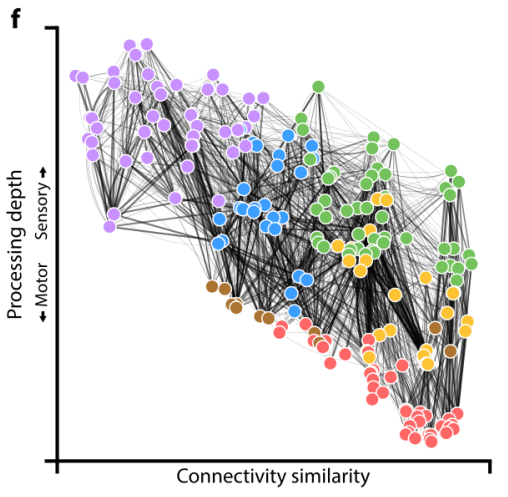

g

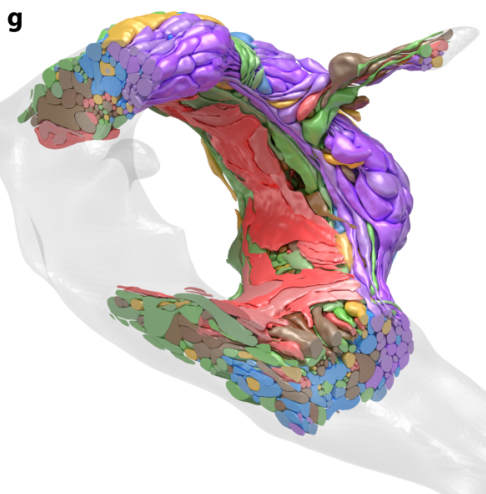

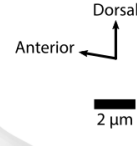

Figure 5. Increase in both feedforward signal flow and modularity across maturation. a. Schematic of feedforward, feedback, and recurrent connections defined by cell types. b. The number of synapses for stable connections in adults (datasets 7 and 8 ) relative to early L1 stages (datasets 1 and 2 ). Stable feedforward connections are strengthened more than stable feedback and recurrent connections. ${ }^{*} \mathrm{p}=4.2 \times 10^{-2},{ }^{\star \star} \mathrm{p}=3.3 \times 10^{-3},{ }^{\star \star \star} \mathrm{p}=1.9 \times 10^{-4}$, Mann-Whitney U test, FDR adjusted using Benjamini-Hochberg correction $\left(n_{\text {feedforward }}=297, n_{\text {recurrent }}=224, n_{\text {feedback }}=114\right)$. Center line, median; box limits, upper and lower quartiles; whiskers, $1.5 x$ interquartile range; outliers not shown. c. Proportions of feedforward, feedback, and recurrent connections for stable and developmentally dynamic connections. ${ }^{* \star} p=3.2 \times 10^{-3},{ }^{* * *} p=9.9 \times 10^{-4}$, two-tailed Z-test of the proportion of feedforward connections, FDR adjusted using Benjamini-Hochberg correction $\left(n_{\text {stable }}=733, n_{\text {added }}=200, n_{\text {weakened }}=18\right)$. d. Proportions of the total number of synapses in feedforward, feedback, and recurrent connections. ns (not significant) $p=8.3 \times 10^{-2},{ }^{*} p=1.1 \times 10^{-2},{ }^{\star *} p=1.1 \times 10^{-3}$, Spearman's rank correlation, FDR adjusted using Benjamini-Hochberg correction. e. The number of cells in each module across maturation, determined by weighted stochastic blockmodeling. Modules connected by a line share significant number of neurons (see Table $\mathrm{S} 2$ for cell membership of each module). $\mathrm{f}$. The wiring diagram for the adult connectome (dataset 8 ), with each cell colored by its assigned module. Cell coordinates are represented as in Fig. 1b. g. A 3D model of the adult brain (dataset 8), with each cell colored by its assigned module.

able connections (Fig. 4b upper panel). Developmentally dynamic connections were also prevalent between many sensory neurons, and from sensory neurons to interneurons and motor neurons (Fig. 4c, Fig. S6).

These findings show that maturation changes how sensory information is integrated and relayed to downstream neurons. Maturation also changes motor execution. However, the layout of interneuron circuits, the core decision-making architecture of the brain, is largely stable from birth to adulthood.

\section{Increase in both feedforward signal flow and modularity across maturation}

With connectomes of complete brains, we were able to ask how the sum of synaptic changes leads to collective changes in information processing across maturation.

First, we examined how synaptic changes affect information flow in the brain. The directionality of signal flow between cells can be viewed as either feedforward, feedback, or recurrent (Fig. 5a). We classified connections that constitute synapses from the sensory to motor layer as feedforward, connections from the motor to sensory layer as feedback, and connections between neurons of the same type as recurrent. Among stable connections, synapse addition strengthened existing feedforward connections more than feedback or recurrent connections (Fig. 5b). The addition of developmentally dynamic connections also preferentially increased feedforward signal flow (Fig. 5c). In contrast, developmentally dynamic connections that were weakened across maturation tended to be feedback and recurrent. Cumulatively, the proportion of synapses that constitute feedforward connections gradually increased (Fig. 5d). Thus, one global pattern of brain maturation augments signal flow from sensation to action, making the brain more reflexive (and less reflective) with age.

Next, we asked how changes in connections affect the community structure of the brain. We used weighted stochastic blockmodeling (WSBM) to group neurons of similar connectivity into distinct modules ${ }^{32}$. We found that the wiring diagram becomes more modular across maturation, increasing from two modules at birth to six modules in adulthood (Fig. 5e, Fig. S8a, Table S2). A similar increase was obtained with a generative evaluation framework, an independent estimator of modularity (Fig. S8b, see Methods). The increase in modularity can be mostly attributed to developmentally dynamic connections, which only represent $12 \%$ of total synapses (Table S3). Variable connections, which are not uniformly distributed among cell types, also contributed to module segregation (Table S3).

Increased modularity produces congregations of cells and circuits with functional specialization. At birth, sensory neurons and interneurons that relay and integrate sensory information were clustered into one module. By adulthood, labial sensory neurons ("anterior sensory"), amphid sensory neurons ("posterior sensory"), and remaining sensory neurons and the majority of interneurons ("medial sensory/interneuron"), became sepa- 


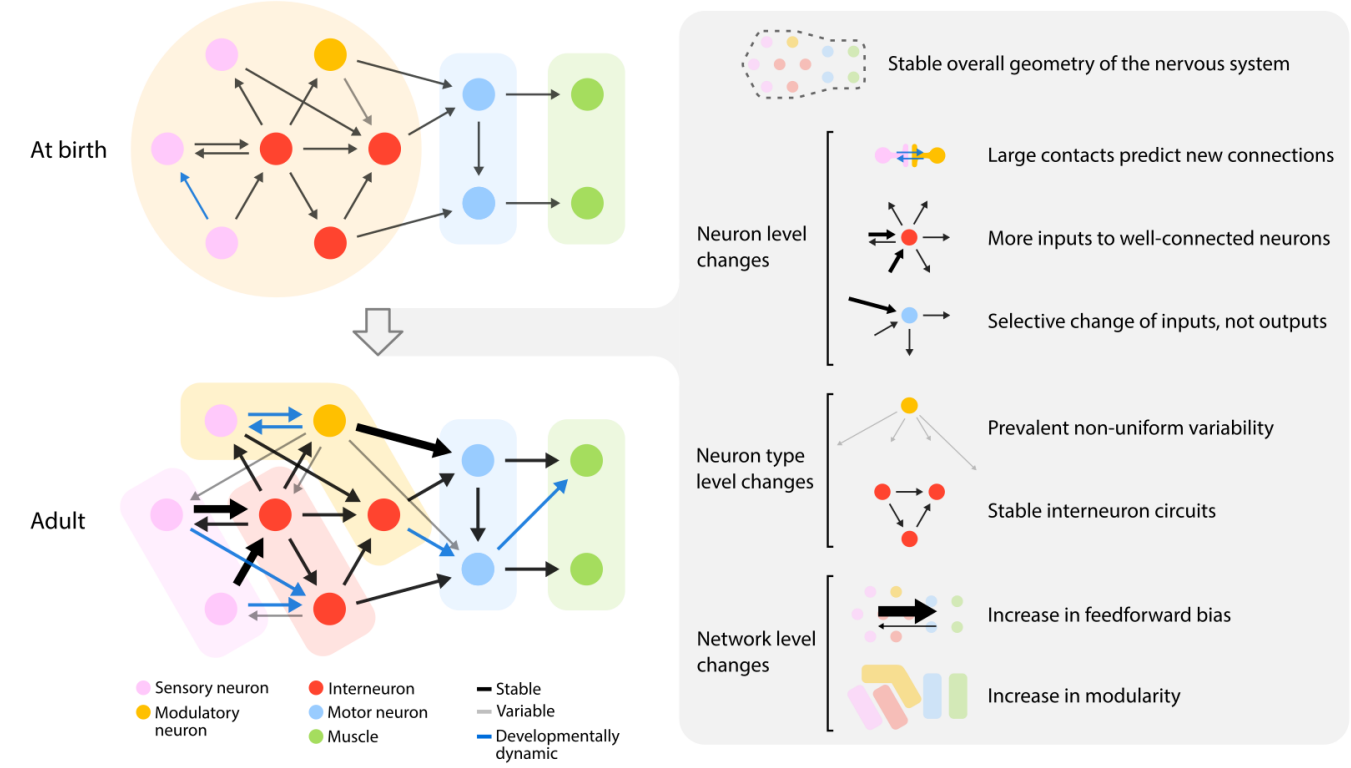

Figure 6. Principles of connectivity changes across maturation. Left: schematic of brain-wide synaptic changes from birth to adulthood. Right: emerging principles of maturation that describe synaptic changes at the level of brain geometry, individual neurons, neuron types, and entire networks.

rate modules (Fig. 5e). At birth, head motor neurons and premotor interneurons that command body movements were clustered into the same module. In adults, they belonged to separate modules ("head movement" and "body movement") (Fig. 5e, Table S2). Importantly, functional modules are created from closely connected neurons in the wiring diagram (Fig. 5f) as well as physically proximate neurons, reminiscent of distinct brain lobes (Fig. 5g, Video 4).

\section{Discussion}

To learn if any principles emerge by studying synaptic changes of an entire brain across maturation, we analysed eight isogenic $C$. elegans beginning with the earliest larva stage and ending with the adult. While it took nearly a decade to assemble the first $C$. elegans connectome ${ }^{25}$, the advent of automation in sample sectioning, image acquisition, and data processing sped up the process, allowing our complete brain reconstructions of multiple animals in far less time.

We found that several general features remained largely unchanged from the earliest larva to the adult stage. For example, the overall geometry of the brain, the three-dimensional shape and relative placement of individual neurons, was surprisingly stable.

In contrast, the total volume of the brain enlarged about 6fold. However, changes in brain connectivity were not simply explained by enlargement of existing structures. While there was a 5 -fold increase in the number of synapses, these synaptic changes were not distributed uniformly through the network. Rather, they appeared to be organized by several developmental principles that profoundly shape how the brain's network changes.

The principles that we uncovered are illustrated in Fig. 6. At one level, we observed patterns of synaptic remodelling that differentially alter the number and strength of connections, applied to every neuron. At a second level, we observed patterns of synaptic remodelling that differ between cell types (i.e., sensory neurons, modulatory neurons, interneurons, and motor neurons). At the third level, we observed network-level changes that alter the directionality of information flow and the segregation of information processing throughout the brain. Likely, these three levels of synaptic remodelling (listed below) explain the ontogenetic basis of adult behaviour:

Large contacts predict new connections. Because the overall geometry of the brain is constant, physical contacts between neurites from birth to adulthood are nearly invariant. Nearly all new synapses appear at sites where these physical contacts already exist, both adding synapses to connections between neurons and creating new connections between neurons. The larger the physical contact, the greater the probability of a new connection. Therefore, the brain's geometry at birth creates the scaffold upon which adult connectivity is built.

More inputs to well-connected neurons. We found that developmental synapse addition was not equal among all neurons. Cells with larger number of connections at early stages receive disproportionately more new synapses, both strengthening existing input connections and creating new input connections. In contrast, these neurons see less synapse addition to their output connections. Thus, well-connected neurons become better integrators of information, but not broader communicators of that information.

Selective change of a neuron's inputs, but not outputs. We also found a pattern in how synapses selectively change the strengths of existing connections. The strength (synapse number) of input connections that converge on the same neuron tends to become more heterogeneous. In contrast, the outputs from the same neuron maintain their relative strengths. Neurons thus become differentially driven by a subset of their presynaptic partners, but distribute that information uniformly among their postsynaptic partners.

Prevalent variability in the connectomes between animals.

Each animal has connections between neurons that were are found in other animals. These variable connections tend to be mediated by small numbers of synapses, but nonetheless represent almost half of all connected neuron pairs in the mature brain. This variability is most prominent among the modulatory neurons and least prominent among motor neurons. 
Stable interneuron circuits. We discovered remarkable stability in the wiring between interneurons that may constitute the core decision-making architecture of the brain. In contrast, we found extensive developmental wiring changes among other cell types.

Increase in feedforward bias. At the level of the entire network, we discovered a change in the directionality of information flow. Synaptogenesis over development preferentially creates new connections and strengthens existing connections in the direction of sensory layers to motor layers. This makes the network more feedforward and reflexive over time.

Increase in modularity. Synaptogenesis also progressively increases the community structure of the brain, as sub-networks for sensory or motor processing gradually emerge across maturation.

These principles have ontogenetic, phylogenetic, and functional implications discussed below.

\section{The C. elegans wiring diagram is not stereotyped}

We found that there is considerable variability in synaptic connectivity between this set of isogenic animals. About $43 \%$ of all connections and $16 \%$ of all synapses are not conserved between animals. This degree of variability contrasts with the view that the C. elegans connectome is hardwired. In C. elegans, the idea that individual neurons have identical connectivities probably stemmed from the fact that individual neurons are identifiable in each animal by virtue of their mostly stereotyped lineage and morphology $25,30,33$. This stereotypy implies that many properties are genetically determined. Hence, if genetic regulation is strong, each identified neuron may have the same connections from one animal to another in an isogenic population. The original connectome, because it was assembled from partial datasets and only one complete adult nerve ring, could not address variability ${ }^{25}$

We found that synaptic variability between animals is not uniform among cell types. For example, modulatory neurons have considerable variability in their output connections whereas motor neurons have little variability in their outputs. This contrast suggests that variability is in some way regulated between cell types, and may therefore be genetically determined and functionally important. For example, behavioural variability between animals can confer a fitness advantage to a population ${ }^{34}$. Synaptic variability may be a source of such behavioural variability, e.g., in the Drosophila visual system, variability among neurite morphologies has been linked to behavioural individuality 35

In isogenic animals, one mechanism for synaptic variability may be stochastic differences in gene expression. Variable expression levels have been observed even in the housekeeping genes in C. elegans embryos ${ }^{36}$. Neuronal activity can also be a driving force for synaptic remodelling. Individuals from an isogenic population reared in similar conditions will still experience differences in their local environments throughout life, a source of differences in neuronal activity that may translate into wiring variability ${ }^{37}$.

\section{Developmental changes in the periphery of the connec-} tome versus constancy in the central core

Why is interneuron connectivity more stable across maturation when compared to the sensory input and motor output of the brain? From an evolutionary standpoint, it may not be surprising that the part of a nervous system that physically interacts with the outside world (mainly sensory systems and motor systems) is under high evolutionary pressure to maintain an animal's fitness in changing environments. Such evolutionary changes in the nematode brain (phylogeny) may have accrued as developmental changes (ontogeny) in its wiring diagram.

Stability of the core parts of the nervous system across maturation implies that the central processing unit is robust enough to be used in different contexts. Maturation changes the flow of sensory information into the central processor and the readout of motor execution from the central processor, without changing the central processor itself. Sensory maturation may reflect changes caused by learning and memory ${ }^{38}$. Motor circuit maturation may reflect adaptations to the changing musculature of the growing animal body ${ }^{39}$.

\section{The connectome becomes more feedforward during mat- uration}

We observed an increased feedforward-bias of the adult brain that may be more effective in rapid information processing and making reflexive decisions. In contrast, the juvenile network with more feedback connections may have a greater capacity for learning and adaptation. Interestingly, feedback is what is used to train some artificial neural networks that perform machine learning ${ }^{40}$. After these artificial networks achieve their desired performance, they operate in a feedforward manner. The architecture of the adult nematode brain may be a consequence of feedback-mediated optimization of its sensorimotor pathways.

\section{The connectome becomes more modular during matura- tion}

We observed an increased community structure of the brain's network that suggests the emergence of specialized circuits for distinct roles. Each functional community emerges among neurons that are physically close to one another (Fig. $5 \mathrm{~g}$ ). In the nematode brain, the physical layout precedes the functional layout. Over time, neurons in proximity are more likely to acquire similar functionality by building their connectivity. The communities that are formed effectively create spatially compact areas for sensory or motor processing, reminiscent of distinct brain areas in larger animals.

\section{Perspectives}

In larger animals that mature more slowly, maturation involves extensive changes in the nervous system. Apoptosis, neurite degeneration, and synapse pruning remove unwanted circuitry ${ }^{41}$. Neurogenesis, neurite growth, and synapse formation create new circuitry ${ }^{42}$. For the short-lived C. elegans, maturation must be fast and efficient. In its small nervous system, each cell is unique, and each is characterized by an intrinsic propensity for synaptic remodelling. These changes occur in the context of its stable morphology and fixed physical contacts with its neighbours. With these constraints, the nematode has evolved a broad set of principles for synaptic maturation to build its adult brain (Fig. 6).

In the C. elegans brain, synaptic remodelling leads to changes from the cell to network level, with likely profound functional consequences on behaviour. Most investigations of flexibility in neural circuits and behaviours focus on functional modulations of connectomes that have been assumed to be anatomi- 
cally static ${ }^{43,44}$. Our comparison of connectomes argues that the maturation and variability of brain and behaviour cannot be separated from wiring changes; moreover, comparative connectomics is needed to understand the origin of similarity and difference in structure and behaviour, within and across species. High-throughput and high-resolution electron microscopy are necessary to establish the foundation for understanding how genes, experience, and evolution create adult brain and behaviour.

\section{Methods}

\section{Data acquisition}

We studied wild-type (Bristol N2) animals reared in standard conditions: 35x10mm NGM-plates, fed by OP50 bacteria, and raised at $22.5^{\circ} \mathrm{C}^{45}$. The animals were within a few generations of the original stock acquired from Caenorhabditis elegans $\mathrm{Ge}$ netics Center (CGC) in 2001. All samples used in this study were derived from three batches of EM preparation.

Each EM sample was prepared and processed as previously described $^{29}$ with small modifications to the substitution protocol of the last 3 datasets (protocol in preparation). In short, isogenic samples reared in the same environment were highpressure frozen (Leica HPM100 for datasets 1-5 and Leica ICE for datasets 6-8) at different stages of post-embryonic development. High-pressure freezing was followed by freezesubstitution in acetone containing $0.5 \%$ glutaraldehyde and $0.1 \%$ tannic acid, followed by $2 \%$ osmium tetroxide. For each life stage, we selected animals based on their overall size and morphology for EM analysis. The precise developmental age of each larval animal was determined based on its cellular compositions relative to its stereotyped cell lineage ${ }^{30}$, as well as the extent of its neurite growth (see Supplemental Text). Three samples (datasets 2, 6, and 7) were prepared for transmission electron microscopy (TEM). Five samples (datasets 1, 3, 4, 5, and 8) were prepared for scanning electron microscopy (SEM).

For TEM, samples were manually sectioned at $\sim 50 \mathrm{~nm}$ using a Leica UC7 ultramicrotome, collected on formvar-coated slot grids (Electron Microscopy Sciences, FF205-Cu), post-stained with $2 \%$ aqueous uranyl acetate and $0.1 \%$ Reynold's lead citrate, and coated with a thin layer of carbon. Images were acquired using an FEI Techai 20 TEM and a Gatan Orius SC100 CCD camera.

For SEM, samples were serial sectioned at $\sim 30 \mathrm{~nm}$ and collected using an automated tape-collecting ultramicrotome (ATUM $)^{46}$. The tape was glued to silicon wafers, carbon coated, and sections post-stained with $0.5 \%$ uranyl acetate (Leica Ultrostain I, Leica Microsystems) and 3\% lead citrate (Leica Ultrostain II, Leica Microsystems). Images were collected semiautomatically using custom software guiding an FEI Magellan XHR 400L ${ }^{47}$.

All images were acquired at $0.64-2 \mathrm{~nm} / \mathrm{px}(\sim 25,000 \mathrm{x})$. In total, these datasets comprise 94374 images, 5 teravoxels, and $2.4 \times 10^{5} \mu^{3}$. Images were aligned using TrakEM2 ${ }^{48,49}$ and imported into CATMAID ${ }^{50}$ for annotation.

\section{Connectome annotation}

All cells within the brain were manually reconstructed by skeleton tracing in CATMAID ${ }^{50}$. The brain was defined as the nerve ring and ventral ganglion neuropil anterior of the ventral sub- lateral commissures. Chemical synapses were mapped manually. To reduce biases from different annotators, all datasets were annotated independently by three different people. Only synapses that were agreed to by at least two independent annotators were included in the final dataset.

Neurons were identified based on cell body position, neurite trajectory, and stereotypic morphological traits ${ }^{25}$. In the original connectome datasets, as well as ours, some variability in cell body position was observed (see Supplemental text). However, every cell could be unambiguously identified in every dataset when all anatomical factors were taken into account. Negligible amounts of neuropil in our reconstructions could not be reliably identified as belonging to any known cell. These orphan fragments were relatively small (median length $0.38 \mu \mathrm{m}$ ) and rare $(4.13 \pm 6.05$ per dataset). Orphan fragments represent $0.18 \%$ of the total neurite length and $0.13 \%$ of all synapses, and were excluded from analysis.

Chemical synapses were identified by a characteristic presynaptic swelling containing a pool of clear vesicles adjacent to a dark active zone on the inside of the membrane ${ }^{29}$. Any cell adjacent to the active zone was identified as a postsynaptic partner. Presynaptic swellings were also typically characterized by mitochondria and cadherin-like junctions between pre- and postsynaptic cells ${ }^{51}$. A small fraction of postsynaptic partners exhibited postsynaptic densities.

Chemical synapses came in two varieties: classical synapses containing mostly clear synaptic vesicles surrounding the active zone and synapses of modulatory neurons containing mostly dense-core vesicles distant from the active zone. Most classical synapses also contained a small number of large dense-core vesicles at the periphery of the vesicle pool. Besides chemical synapses, neurons contained swellings with vesicles but no active zones. The majority of swellings of modulatory neurons did not have active zones ${ }^{52}$. These swellings were not annotated as synapses.

Final synapse annotations for all datasets are available at http: //nemanode.org/.

\section{Classification of neuron types}

Neurons were classified as modulatory if they contained mostly large dark vesicles, or if they had been previously reported to use the neurotransmitters serotonin, dopamine, or octopamine ${ }^{53,54}$. Neurons were classified as motor neurons if they primarily made synapses onto muscles. Neurons were classified as sensory if they had specialized sensory processes and/or were previously reported to have sensory capabilities. Neurons were classified as interneurons if most of their connections were to other neurons. Some neurons exhibit features corresponding to more than one type. These neurons were classified based on their most prominent feature (Table S1).

\section{Segmentation for volumetric reconstruction}

We computed the precise shape of every neurite in each EM image based on the skeleton tracing that was performed in CATMAID and a machine learning algorithm that recognized cellular boundaries. In brief, the algorithm expanded all skeleton nodes in each section until they fully filled the images of all labelled cells.

Cellular borders were predicted by a shallow Convolutional Neural Network $(\mathrm{CNN})$ that builds on $X N N^{55,56}$, a recently 
developed high performance system which computes convolutions on CPUs, to achieve border prediction throughput of $\sim 10 \mathrm{MB} / \mathrm{s}^{57,58}$. Node expansion was computed with a dedicated Cilk-based code ${ }^{59}$ that parallelized the Dijkstra graph search algorithm. Code optimization allowed us to perform node expansion of an entire EM section in memory by a single multithreaded process. Each software thread expanded an individual skeleton. Each pixel is attributed to a given cell by computing a generalized form of distance, taking into account the minimum number of cellular border pixels that must be traversed in a path connecting pixel and node. The generalized distance is computed using graph theory and concurrent data structures.

Volume traces were imported into VAST ${ }^{60}$ for manual proofreading. At least 1,120 person-hours were spent proofreading the volumetric expansions. Volumetric reconstruction was not performed on dataset 7 due to weak membrane contrast.

\section{Data processing for analysis}

Volumetric neuron traces were exported from VAST ${ }^{60}$ and imported into MATLAB. EM artefacts were manually corrected. To calculate the contact area of each adjacent cell pair, we performed two-dimensional morphological dilation of every traced segment across extracellular space until neighbouring segments made contact within 70 pixels (140-280nm). Expansion was restricted to the edge of the nerve ring. The total contact area was calculated as the sum of adjacent pixels for each segment in all sections. Contacts between cell bodies at the periphery of the neuropil were excluded.

Neuron skeletons and synapses were exported from CATMAID using custom Python scripts, and imported into Python or MATLAB environments for analyses. The module detection analysis was performed in MATLAB. Other analyses were implemented with custom Python scripts using SciPy and Statsmodels libraries for statistics. Post-embryonically born neurons were excluded for analysis related classification of connections, feedforward information flow, and modulatory.

For analyses related to neurites, both processes of neurons and muscles in the nerve ring were included. The neurite length was calculated using the smoothened skeleton of each neurite. The skeleton was smoothed by a moving average of 10 skeleton nodes after correction of major alignment shifts. Spine-like protrusions were defined as any branch shorter than the $10 \%$ of the average neuron length. For analyses related to information flow, separating connections into feedforward, feedback, and recurrent, connections to muscles were excluded since they are all feedforward.

\section{Classification of connections}

A total of 3113 connections (averaging 1292 per dataset) were assigned as stable, variable, or developmentally dynamic. 1647 weak connections (averaging 323 per dataset) had no more than two synapses in two or more datasets and were left-right asymmetric. These connections were classified as variable. The 1466 remaining connections were pooled by left-right cell pairs, resulting in 658 pair connections. The number of synapses in each pair connection was tested for relative increase or decrease across maturation (Spearman's rank correlation, corrected for multiple comparisons using the Benjamini-Hochberg correction). Pair connections showing a significant change and at least a 5-fold change in synapse number from birth to adulthood were classified as developmentally dynamic. Remaining pair connections were considered stable if they were present in at least 7 datasets, and variable if present in fewer than 7 datasets.

\section{Comparison to the original $C$. elegans adult connectome}

The original adult hermaphrodite brain connectome annotated by White et al. ${ }^{25,61}$ was taken from wormatlas.org (dataset $\mathrm{N} 2 \mathrm{U}$ ). Individual muscles were not traced in the original connectome. We completed this dataset by manually tracing all the head muscles using the scanned EM micrographs hosted by wormatlas.org. This dataset (referred to as N2U, White et al., 1986) was used for subsequent analysis.

The reannotated $\mathrm{N} 2 \mathrm{U}$ connectome by Cook et al. ${ }^{22}$ was taken from wormwiring.org (referred to as N2U, Cook et al., 2019). We only included neurons and neurites within the same regions used for our datasets and N2U, White et al., 1986. In addition, we corrected several errors in their muscle identification in the published dataset. Specifically, we switched the muscle pairs VL1-VL2, VR1-VR2, DL2-DL3, DR2-DR3, DL5-DL6, DR5DR6, VL5-VL6, and VR5-VR6. Other mistakes in their tracing and synapse annotation could not be corrected for. For example, muscles DL7 and DL8 were missing entirely in the brain, and only one of more than 50 synapses onto muscle VR2 (named as VR1 by Cook et al.) was annotated.

\section{Community structure analysis}

Weighted stochastic blockmodeling (WSBM) ${ }^{32}$ was used to define modules individually for all eight connectomes. In this approach, modules are optimized on the likelihood of observing the actual network from the determined modules (log-likelihood score) based on two exponential family distributions. We chose the probability of establishing connections to follow a Bernoulli distribution and the synapse number for each connection to follow an exponential distribution. These distributions fit the data best according to the log-likelihood score and resulted in leftright cell pairs being assigned to the same modules.

In order to find a stable and representative number of modules for each connectome, we used a consensus-based modelfitting approach, similar to previously described ${ }^{62}$. First, to ensure unbiased coverage of the parameter space, we fitted the model independently 300 times using an uninformative prior for each potential number of modules $(\mathrm{k}=1, \ldots, 10)$. This procedure was repeated 100 times to yield a collection of models with concentrated and unimodally distributed log evidence scores. To improve the stability of the models on multiple runs, we increased the parameters for a maximum number of internal iterations to 100 . For each dataset, we chose the number of modules whose collection of models had the highest mean posterior log-likelihood score. If scores for two different numbers of modules had significant overlap, the number of modules closest to the connectome at an earlier developmental timepoint was selected.

Finally, for each dataset, we found a representative consensus module assignment that summarized all 100 models ${ }^{62}$. In brief, considering all 100 models, we calculated the frequency of each cell being assigned to each module, and used this as a new prior to fit another 100 models. This procedure was repeated until convergence, when the consistency of the 100 models was larger than 0.95 . 
bioRxiv preprint doi: https://doi.org/10.1101/2020.04.30.066209; this version posted May 22, 2020. The copyright holder for this preprint (which was not certified by peer review) is the author/funder, who has granted bioRxiv a license to display the preprint in perpetuity. It is made available under aCC-BY-NC-ND 4.0 International license.

\section{Community structure validation}

We validated the community structure defined by WSBM using a previously described method ${ }^{62}$. In brief, for each possible number of modules $\mathrm{k}=1, \ldots, 10$, the quality of the best final model determined by WSBM was examined to validate the model chosen by the log-likelihood score. For each k, we fit a WSBM model with a prior matching the module assignment, and reverse simulated 2000 synthetic connectomes from the model. For each synthetic connectome, we recorded 8 statistic measurements: degree distribution, in-degree distribution, outdegree distribution, weight distribution, in-weight distribution, out-weight distribution, betweenness centrality, and weighted clustering coefficient. These distributions were compared to the actual connectome using a Kolmogorov-Smirnov (KS) statistic test, and summarized by computing the mean KS energy, defined as the mean value of all $8 \mathrm{KS}$ statistic values. A lower mean KS energy indicated a better match. For the connectomes of early developmental stages, an equal match was found for $\mathrm{k}$ $=3 \ldots 6$ (Fig. S8b). For the adult connectomes, $\mathrm{k}=6$ matched the connectome significantly better than $\mathrm{k}<6$ (Fig. S8b).

\section{Statistics}

Statistical methods were not used to predetermine sample sizes. Spearman's rank was used for all correlations (Fig. 2d-2f, S3b, S8c and S8e) and time series (Fig. 2g, 3, S3c-S3e and 5d). Twotailed Z-test was used to compare proportions (Fig. 4c, 5c). To determine if developmentally dynamic connections were overor underrepresented, the proportions of developmentally dynamic connections between each cell type were compared to the total proportion of developmentally dynamic connections throughout all cell types (Fig. 4c). Kruskal-Wallis test followed by pairwise Mann-Whitney U tests were used for comparisons of more than two unpaired categories (Fig. 4b, 5b, S5b and S8a). For figure panels with more than three categories, only categories statistically different from all others were labelled (Fig. 4b, S8a. For figure panels with multiple comparisons, the reported p-values were FDR adjusted using Benjamini-Hochberg correction.

\section{Data availability}

All electron microscopy images, volumetric reconstructions, and skeleton reconstructions will be made available on a public repository before publication. Connectivity matrices for all datasets are available on www.nemanode.org and as supplementary data.

\section{Data and code availability}

Scripts used to generate all figures will be made available on a public repository before publication.

\section{ACKNOWLEDGEMENTS}

We thank Valeriya Laskova for assistance in developing the EM sample preparation protocol. We thank Bob Harris for assistance with high-pressure freezing. We thank Marianna Neubauer, David Kersen, Anabelle Paulino, Manusnan Suriyalaksh, Amelia Srajer, Maggie Chang, Sean Ihn, and Jade Ho for help with imaging. We thank Albert Cardona, Ignacio Arganda-Carreras and Jenny Qian for guidance on with EM alignment. We thank Steven Cook, Christine Rehaluk, and Mona Wang for synapse annotation in some datasets. We thank Jade Ho, Christopher Morii-Sciolla, Isis So, Min $\mathrm{Wu}$ and Chi Yip Ho for help with generating ground truth and proofreading for volumetric reconstruction. We thank Alexander Matveev, Lu Mi, and Hayk Saribekyan for help generating and applying algorithms used in this project. We thank Jerry Wang and Danqian Cao for help with statistical analyses. We thank Albert Lin, Chris Tabone, and Vivek Venkatachalam for setting up and supporting the server for synapse annotation. We thank Soomin Maeng and Dylan Fong for assistance with the develop of www.nemanode.org. We thank members of the Zhen, Samuel, and Lichtman labs for comments. We especially thank Guangwei Si and Lav Varsney for critical reading and suggestions. We thank David Hall, Jagan Srinivasan, and Albert Cardona for early advice in this project.

J.K.M. was supported by National Science Foundation Physics of Living Systems (NSF 1806818). B.M. was supported by the Mount Sinai Foundation. J.W.L. was supported by the National Institute of Mental Health, Silvio Conte Center (P50 MH094271), the National Institutes of Health (U24 NS109102-01), and the Multidisciplinary University Research Initiative (GG0008784). J.W.L., A.D.T.S., and M.Z. were supported by the Human Frontier Science Program (RGP0051/2014). A.D.T.S and M.Z. were supported by the National Institutes of Health (R01-NS082525-01A1). A.D.T.S. was supported by National Institutes of Health Brain Initiative (1U01NS111697-01) and National Science Foundation BRAIN EAGER (IOS-1452593). M.Z. was supported by Canadian Institutes of Health Research (MOP-123250 and Foundation Scheme 154274), the Radcliffe Institute, and the Mount Sinai Foundation.

\section{AUTHOR CONTRIBUTIONS}

J.W.L, A.D.T.S., and M.Z. conceived the study. Y.M., R.P., and N.S. designed the algorithm for automated volumetric reconstruction (yaron.mr@gmail.com for correspondence). D.R.B. and R.L.S. designed the pipeline for automated EM acquisition (danielberger@fas.harvard.edu for correspondence). Y.W. designed software for EM alignment (yuelongwu@fas.harvard.edu for correspondence). D.W., B.M., J.K.M., D.H., R.L.S, and M.Z. generated and imaged most of the electron micrographs. D.W., B.M., and J.K.M. performed most annotation. D.W. performed most analysis. D.R.B., W.X.K., and Y.L. performed additional experiments and analysis. A.D.C. guided early cell identification and annotation. D.W., J.W.L, A.D.T.S., and M.Z. wrote the manuscript. All authors reviewed the manuscript.

\section{COMPETING INTERESTS}

The authors declare no competing interests.

\section{References}

[1] N.-W. Tien and D. Kerschensteiner. Homeostatic plasticity in neural development. Neural Development, 13(1), Dec. 2018. doi: 10.1186/s13064-018-0105-x.

[2] D. Bucher. Animal-to-Animal Variability in Motor Pattern Production in Adults and during Growth. Journal of Neuroscience, 25(7):1611-1619, Feb. 2005. doi: 10.1523/JNEUROSCI.3679-04. 2005.

[3] G. Kämper and R. Murphey. Maturation of an insect nervous system: Constancy in the face of change. Comparative Biochemistry and Physiology Part A: Physiology, 109(1):23-32, Sept. 1994. doi: 10.1016/0300-9629(94)90308-5.

[4] S. Gerhard, I. Andrade, R. D. Fetter, A. Cardona, and C. M. Schneider-Mizell. Conserved neural circuit structure across Drosophila larval development revealed by comparative connectomics. eLife, 6, Oct. 2017. doi: 10.7554/eLife.29089.

[5] J. Kagan, N. Herschkowitz, and E. C. Herschkowitz. A Young mind in a growing brain. Lawrence Erlbaum, Mahwah, NJ, 2005. ISBN 978-0-8058-5425-1 978-0-8058-5309-4. OCLC: 845860192.

[6] W. Fox. Reflex-ontogeny and behavioural development of the mouse. Animal Behaviour, 13 (2-3):234-IN5, Apr. 1965. doi: 10.1016/0003-3472(65)90041-2.

[7] A. Pujala and M. Koyama. Chronology-based architecture of descending circuits that underlie the development of locomotor repertoire after birth. eLife, 8, Feb. 2019. doi: 10.7554/eLife. 42135 .

[8] D. O. Hebb. The organization of behavior: a neuropsychological theory. John Wiley Amp Sons, Inc., 1949.

[9] B. M. Hooks and C. Chen. Distinct Roles for Spontaneous and Visual Activity in Remodeling of the Retinogeniculate Synapse. Neuron, 52(2):281-291, Oct. 2006. doi: 10.1016/j.neuron.2006. 07.007.

[10] H. Jaaro-Peled, A. Hayashi-Takagi, S. Seshadri, et al. Neurodevelopmental mechanisms of schizophrenia: understanding disturbed postnatal brain maturation through neuregulin-1-ErbB4 and DISC1. Trends in Neurosciences, 32(9):485-495, Sept. 2009. doi: 10.1016/j.tins.2009.05. 007.

[11] S. A. McMahon and E. Díaz. Mechanisms of excitatory synapse maturation by trans-synaptic organizing complexes. Current Opinion in Neurobiology, 21(2):221-227, Apr. 2011. doi: 10. 1016/j.conb.2010.12.005.

[12] M. R. Van Horn and E. S. Ruthazer. Glial regulation of synapse maturation and stabilization in the developing nervous system. Current Opinion in Neurobiology, 54:113-119, Feb. 2019. doi: 10.1016/j.conb.2018.10.002.

[13] K. L. Briggman and D. D. Bock. Volume electron microscopy for neuronal circuit reconstruction. Current Opinion in Neurobiology, 22(1):154-161, Feb. 2012. doi: 10.1016/j.conb.2011.10.022.

[14] L. K. Scheffer, C. S. Xu, M. Januszewski, et al. A Connectome and Analysis of the Adult Drosophila Central Brain. preprint, bioRxiv, Apr. 2020.

[15] A. Karimi, J. Odenthal, F. Drawitsch, K. M. Boergens, and M. Helmstaedter. Cell-type specific innervation of cortical pyramidal cells at their apical dendrites. eLife, 9:e46876, Feb. 2020. doi: 10.7554/eLife.46876.

[16] K. Eichler, F. Li, A. Litwin-Kumar, et al. The complete connectome of a learning and memory centre in an insect brain. Nature, 548(7666):175-182, Aug. 2017. doi: 10.1038/nature23455.

[17] J. L. Morgan and J. W. Lichtman. An Individual Interneuron Participates in Many Kinds of Inhibition and Innervates Much of the Mouse Visual Thalamus. Neuron, page S0896627320301008, Mar. 2020. doi: 10.1016/j.neuron.2020.02.001.

[18] N. Randel, R. Shahidi, C. Verasztó, et al. Inter-individual stereotypy of the Platynereis larval visual connectome. eLife, 4, June 2015. doi: 10.7554/eLife.08069.

[19] J. Kornfeld, M. Januszewski, P. Schubert, et al. An anatomical substrate of credit assignment in reinforcement learning. preprint, bioRxiv, Feb. 2020

[20] C. M. Schneider-Mizell, A. L. Bodor, F. Collman, et al. Chandelier cell anatomy and function reveal a variably distributed but common signal. preprint, bioRxiv, Apr. 2020.

[21] D. J. Bumbarger, M. Riebesell, C. Rödelsperger, and R. J. Sommer. System-wide Rewiring Underlies Behavioral Differences in Predatory and Bacterial-Feeding Nematodes. Cell, 152 (1-2):109-119, Jan. 2013. doi: 10.1016/j.cell.2012.12.013.

[22] S. J. Cook, T. A. Jarrell, C. A. Brittin, et al. Whole-animal connectomes of both Caenorhabditis elegans sexes. Nature, 571(7763):63-71, July 2019. doi: 10.1038/s41586-019-1352-7. 
bioRxiv preprint doi: https://doi.org/10.1101/2020.04.30.066209; this version posted May 22, 2020. The copyright holder for this preprint (which was not certified by peer review) is the author/funder, who has granted bioRxiv a license to display the preprint in perpetuity. It is made available under aCC-BY-NC-ND 4.0 International license.

[23] J. Valdes-Aleman, R. D. Fetter, E. C. Sales, et al. Synaptic specificity is collectively determined by partner identity, location and activity. preprint, bioRxiv, July 2019.

[24] A. M. Wilson, R. Schalek, A. Suissa-Peleg, et al. Developmental Rewiring between Cerebellar Climbing Fibers and Purkinje Cells Begins with Positive Feedback Synapse Addition. Cell Reports, 29(9):2849-2861.e6, Nov. 2019. doi: 10.1016/j.celrep.2019.10.081.

[25] J. G. White, E. Southgate, J. N. Thomson, and S. Brenner. The structure of the nervous system of the nematode Caenorhabditis elegans. Philosophical Transactions of the Royal Society of London. Series B, Biological Sciences, 314(1165):1-340, Nov. 1986

[26] L. R. Varshney, B. L. Chen, E. Paniagua, D. H. Hall, and D. B. Chklovskii. Structural Properties of the Caenorhabditis elegans Neuronal Network. PLoS Computational Biology, 7(2):e1001066, Feb. 2011. doi: 10.1371/journal.pcbi.1001066.

[27] R. M. Weimer. Preservation of $C$. elegans Tissue Via High-Pressure Freezing and FreezeSubstitution for Ultrastructural Analysis and Immunocytochemistry. In C. elegans, volume 351 pages 203-222. Humana Press, New Jersey, Aug. 2006. ISBN 978-1-59745-151-2. doi: 10 1385/1-59745-151-7:203.

[28] R. Schalek, N. Kasthuri, K. Hayworth, et al. Development of High-Throughput, High-Resolution 3D Reconstruction of Large-Volume Biological Tissue Using Automated Tape Collection Ultramicrotomy and Scanning Electron Microscopy. Microscopy and Microanalysis, 17(S2):966-967, July 2011. doi: 10.1017/S1431927611005708.

[29] B. Mulcahy, D. Witvliet, D. Holmyard, et al. A pipeline for volume electron microscopy of the Caenorhabditis elegans nervous system. Frontiers in Neural Circuits, 12:94, 2018. doi: 10. 3389/fncir.2018.00094.

[30] J. E. Sulston and H. R. Horvitz. Post-embryonic cell lineages of the nematode, Caenorhabditis elegans. Developmental biology, 56(1):110-156, Mar. 1977.

[31] A. Philbrook, S. Ramachandran, C. M. Lambert, et al. Neurexin directs partner-specific synaptic connectivity in C. elegans. eLife, 7:e35692, July 2018. doi: 10.7554/eLife. 35692.

[32] C. Aicher, A. Z. Jacobs, and A. Clauset. Learning latent block structure in weighted networks. Journal of Complex Networks, 3(2):221-248, June 2015. doi: 10.1093/comnet/cnu026.

[33] J. E. Sulston, E. Schierenberg, J. G. White, and J. N. Thomson. The embryonic cell lineage of the nematode Caenorhabditis elegans. Developmental biology, 100(1), Nov. 1983.

[34] J. G. Burns and A. G. Dyer. Diversity of speed-accuracy strategies benefits social insects. Current Biology, 18(20):R953-R954, Oct. 2008. doi: 10.1016/j.cub.2008.08.028.

[35] G. A. Linneweber, M. Andriatsilavo, S. B. Dutta, et al. A neurodevelopmental origin of behaviora individuality in the drosophila visual system. Science, 367(6482):1112-1119, 2020. doi: 10 1126/science.aaw7182.

[36] A. Raj, S. A. Rifkin, E. Andersen, and A. van Oudenaarden. Variability in gene expression underlies incomplete penetrance. Nature, 463(7283):913-918, Feb. 2010. doi: 10.1038/nature08781.

[37] J. Akhund-Zade, S. Ho, C. O'Leary, and B. de Bivort. The effect of environmental enrichment on behavioral variability depends on genotype, behavior, and type of enrichment. preprint, bioRxiv, Feb. 2019.

[38] X. Jin, N. Pokala, and C. I. Bargmann. Distinct Circuits for the Formation and Retrieval of an Imprinted Olfactory Memory. Cell, 164(4):632-643, Feb. 2016. doi: 10.1016/j.cell.2016.01.007.

[39] J. G. White, D. G. Albertson, and M. A. Anness. Connectivity changes in a class of motoneurone during the development of a nematode. Nature, 271(5647):764-766, Feb. 1978.

[40] D. E. Rumelhart, G. E. Hinton, and R. J. Williams. Learning representations by back-propagating errors. Nature, 323(6088):533-536, Oct. 1986. doi: 10.1038/323533a0.

[41] M. J. Geden and M. Deshmukh. Axon degeneration: context defines distinct pathways. Current Opinion in Neurobiology, 39:108-115, Aug. 2016. doi: 10.1016/j.conb.2016.05.002.

[42] J. R. Sanes and M. Yamagata. Many Paths to Synaptic Specificity. Annual Review of Cell and Developmental Biology, 25(1):161-195, Nov. 2009. doi: 10.1146/annurev.cellbio.24.110707. 175402.

[43] C. I. Bargmann and E. Marder. From the connectome to brain function. Nature Methods, 10(6): 483-490, June 2013. doi: $10.1038 /$ nmeth.2451.

[44] E. Marder. Neuromodulation of Neuronal Circuits: Back to the Future. Neuron, 76(1):1-11, Oct. 2012. doi: 10.1016/..neuron.2012.09.010.

[45] S. Brenner. The genetics of Caenorhabditis elegans. Genetics, 77(1):71-94, May 1974

[46] V. Baena, R. L. Schalek, J. W. Lichtman, and M. Terasaki. Serial-section electron microscopy using automated tape-collecting ultramicrotome (ATUM). Methods in Cell Biology, 152:41-67, 2019. doi: 10.1016/bs.mcb.2019.04.004.

[47] K. J. Hayworth, J. L. Morgan, R. Schalek, et al. Imaging ATUM ultrathin section libraries with WaferMapper: a multi-scale approach to EM reconstruction of neural circuits. Frontiers in Neural Circuits, 8, June 2014. doi: 10.3389/fncir.2014.00068.

[48] A. Cardona, S. Saalfeld, J. Schindelin, et al. TrakEM2 software for neural circuit reconstruction PloS One, 7(6):e38011, 2012. doi: 10.1371/journal.pone.0038011.

[49] S. Saalfeld, R. Fetter, A. Cardona, and P. Tomancak. Elastic volume reconstruction from series of ultra-thin microscopy sections. Nature Methods, 9(7):717-720, July 2012. doi: 10.1038/ nmeth.2072.

[50] S. Saalfeld, A. Cardona, V. Hartenstein, and P. Tomancak. CATMAID: collaborative annotation toolkit for massive amounts of image data. Bioinformatics, 25(15):1984-1986, Aug. 2009. doi: 10.1093/bioinformatics/btp266.

[51] W. L. Hung, C. Hwang, S. Gao, et al. Attenuation of insulin signalling contributes to FSN-1mediated regulation of synapse development. The EMBO Journal, 32(12):1745-1760, May 2013. doi: 10.1038/emboj.2013.91.

[52] M. A. Lim, J. Chitturi, V. Laskova, et al. Neuroendocrine modulation sustains the C. elegans forward motor state. eLife, 5:e19887, Nov. 2016. doi: 10.7554/eLife.19887.

[53] J. Sulston, M. Dew, and S. Brenner. Dopaminergic neurons in the nematode Caenorhabditis elegans. The Journal of Comparative Neurology, 163(2):215-226, Sept. 1975. doi: 10.1002/ cne. 901630207.

[54] J. S. Duerr, D. L. Frisby, J. Gaskin, et al. The cat-1 Gene of Caenorhabditis elegans Encodes a Vesicular Monoamine Transporter Required for Specific Monoamine-Dependent Behaviors. The Journal of Neuroscience, 19(1):72-84, Jan. 1999. doi: 10.1523/JNEUROSCI.19-01-00072.1999.

[55] N. Kasthuri, K. J. Hayworth, D. R. Berger, et al. Saturated Reconstruction of a Volume of Neocortex. Cell, 162(3):648-661, July 2015. doi: 10.1016/j.cell.2015.06.054.

[56] Y. Meirovitch, A. Matveev, H. Saribekyan, et al. A multi-pass approach to large-scale connectomics. arXiv preprint arXiv:1612.02120, 2016

[57] A. Matveev, Y. Meirovitch, H. Saribekyan, et al. A multicore path to connectomics-on-demand. In Proceedings of the 22nd ACM SIGPLAN Symposium on Principles and Practice of Paralle Programming, pages 267-281, 2017.

[58] Y. Meirovitch, L. Mi, H. Saribekyan, et al. Cross-classification clustering: An efficient multi-object tracking technique for 3-d instance segmentation in connectomics. In Proceedings of the IEEE Conference on Computer Vision and Pattern Recognition, pages 8425-8435, 2019.

[59] R. D. Blumofe, C. F. Joerg, B. C. Kuszmaul, et al. Cilk: An efficient multithreaded runtime system. Journal of parallel and distributed computing, 37(1):55-69, 1996.

[60] D. R. Berger, H. S. Seung, and J. W. Lichtman. VAST (Volume Annotation and Segmentation Tool): Efficient Manual and Semi-Automatic Labeling of Large 3d Image Stacks. Frontiers in Neural Circuits, 12:88, 2018. doi: 10.3389/fncir.2018.00088.

[61] R. M. Durbin. Studies on the development and organisation of the nervous system of Caenorhabditis elegans. University of Cambridge UK, 1987.

[62] J. Faskowitz, X. Yan, X.-N. Zuo, and O. Sporns. Weighted Stochastic Block Models of the Human Connectome across the Life Span. Scientific Reports, 8(1), Dec. 2018. doi: 10.1038/ s41598-018-31202-1. 
bioRxiv preprint doi: https://doi.org/10.1101/2020.04.30.066209; this version posted May 22, 2020. The copyright holder for this preprint (which was not certified by peer review) is the author/funder, who has granted bioRxiv a license to display the preprint in perpetuity. It is made available under aCC-BY-NC-ND 4.0 International license.

\section{Supplemental figures}

Video 1. Fly-through of an adult EM dataset.

Video 2. Volumetric reconstruction of an adult dataset.

Video 3. Individual neurons across maturation.

Video 4. Modules in the adult brain. 
Table S1. Cell types in the nerve ring.

\begin{tabular}{|c|c|c|c|}
\hline Class & Members & Type & $\begin{array}{l}\text { Integration into } \\
\text { nerve ring }\end{array}$ \\
\hline $\mathrm{ADA}$ & 2 & inter & embryonic \\
\hline ADE & 2 & modulatory & embryonic \\
\hline ADF & 2 & sensory & embryonic \\
\hline ADL & 2 & sensory & embryonic \\
\hline AFD & 2 & sensory & embryonic \\
\hline AIA & 2 & inter & embryonic \\
\hline AIB & 2 & inter & embryonic \\
\hline AIM & 2 & modulatory & embryonic \\
\hline AIN & 2 & inter & embryonic \\
\hline AIY & 2 & inter & embryonic \\
\hline AIZ & 2 & inter & embryonic \\
\hline ALA & 1 & modulatory & embryonic \\
\hline ALM & 2 & sensory & embryonic \\
\hline ALN & 2 & sensory & post-embryonic \\
\hline AQR & 1 & sensory & post-embryonic \\
\hline ASE & 2 & sensory & embryonic \\
\hline ASG & 2 & sensory & embryonic \\
\hline ASH & 2 & sensory & embryonic \\
\hline ASI & 2 & sensory & embryonic \\
\hline ASJ & 2 & sensory & embryonic \\
\hline ASK & 2 & sensory & embryonic \\
\hline AUA & 2 & sensory & embryonic \\
\hline AVA & 2 & inter & embryonic \\
\hline AVB & 2 & inter & embryonic \\
\hline AVD & 2 & inter & embryonic \\
\hline AVE & 2 & inter & embryonic \\
\hline AVF & 2 & modulatory & post-embryonic \\
\hline AVH & 2 & modulatory & embryonic \\
\hline AVJ & 2 & modulatory & embryonic \\
\hline AVK & 2 & modulatory & embryonic \\
\hline AVL & 1 & modulatory & embryonic \\
\hline AVM & 1 & sensory & post-embryonic \\
\hline AWA & 2 & sensory & embryonic \\
\hline AWB & 2 & sensory & embryonic \\
\hline AWC & 2 & sensory & embryonic \\
\hline $\mathrm{BAG}$ & 2 & sensory & embryonic \\
\hline $\mathrm{BDU}$ & 2 & inter & embryonic \\
\hline BWM01 & 4 & muscle & embryonic \\
\hline BWM02 & 4 & muscle & embryonic \\
\hline BWM03 & 4 & muscle & embryonic \\
\hline BWM04 & 4 & muscle & embryonic \\
\hline BWM05 & 4 & muscle & embryonic \\
\hline BWM06 & 4 & muscle & embryonic \\
\hline BWM07 & 4 & muscle & embryonic \\
\hline BWM08 & 4 & muscle & embryonic \\
\hline
\end{tabular}

\begin{tabular}{|l|l|l|l|}
\hline Class & Members & Type & $\begin{array}{l}\text { Integration into } \\
\text { nerve ring }\end{array}$ \\
\hline CEP & 4 & modulatory & embryonic \\
\hline CEPsh & 4 & glia & embryonic \\
\hline DVA & 1 & sensory & embryonic \\
\hline DVC & 1 & inter & embryonic \\
\hline FLP & 2 & sensory & embryonic \\
\hline GLR & 6 & glia & embryonic \\
\hline HSN & 2 & modulatory & post-embryonic \\
\hline IL1 & 6 & motor & embryonic \\
\hline IL2 & 6 & sensory & embryonic \\
\hline OLL & 2 & sensory & embryonic \\
\hline OLQ & 4 & sensory & embryonic \\
\hline PLN & 2 & sensory & post-embryonic \\
\hline PVC & 2 & inter & embryonic \\
\hline PVN & 2 & inter & post-embryonic \\
\hline PVP & 2 & inter & embryonic \\
\hline PVQ & 2 & modulatory & embryonic \\
\hline PVR & 1 & inter & embryonic \\
\hline PVT & 1 & inter & embryonic \\
\hline RIA & 2 & inter & embryonic \\
\hline RIB & 2 & inter & embryonic \\
\hline RIC & 2 & modulatory & embryonic \\
\hline RID & 1 & modulatory & embryonic \\
\hline RIF & 2 & inter & embryonic \\
\hline RIG & 2 & inter & embryonic \\
\hline RIH & 1 & inter & embryonic \\
\hline RIM & 2 & inter & embryonic \\
\hline RIP & 2 & inter & embryonic \\
\hline RIR & 1 & inter & embryonic \\
\hline RIS & 1 & modulatory & embryonic \\
\hline RIV & 2 & motor & embryonic \\
\hline RMD & 6 & motor & embryonic \\
\hline RME & 4 & motor & embryonic \\
\hline RMF & 2 & motor & post-embryonic \\
\hline RMG & 2 & modulatory & embryonic \\
\hline RMH & 2 & motor & post-embryonic \\
\hline SAA & 4 & sensory & embryonic \\
\hline SDQ & 2 & embryonic & post-embryonic \\
\hline SIA & 4 & embryonic \\
\hline SIB & 4 & embryonic \\
\hline SMB & 4 & embryonic \\
\hline SMD & 4 & embryonic \\
\hline URA & 4 & motor \\
\hline URB & 2 & embryonic \\
\hline URX & 2 & embryonic \\
\hline URY & 4 & 1 & \\
\hline excgl & & & emory \\
\hline
\end{tabular}


bioRxiv preprint doi: https://doi.org/10.1101/2020.04 30.066209; this version posted May 22, 2020. The copyright holder for this preprint (which was not certified by peer review) is the author/funder, who has granted bioRxiv a license to display the preprint in perpetuity. It is made available under aCC-BY-NC-ND 4.0 International license.

Table S2. Members of modules detected by WSBM colored by type.

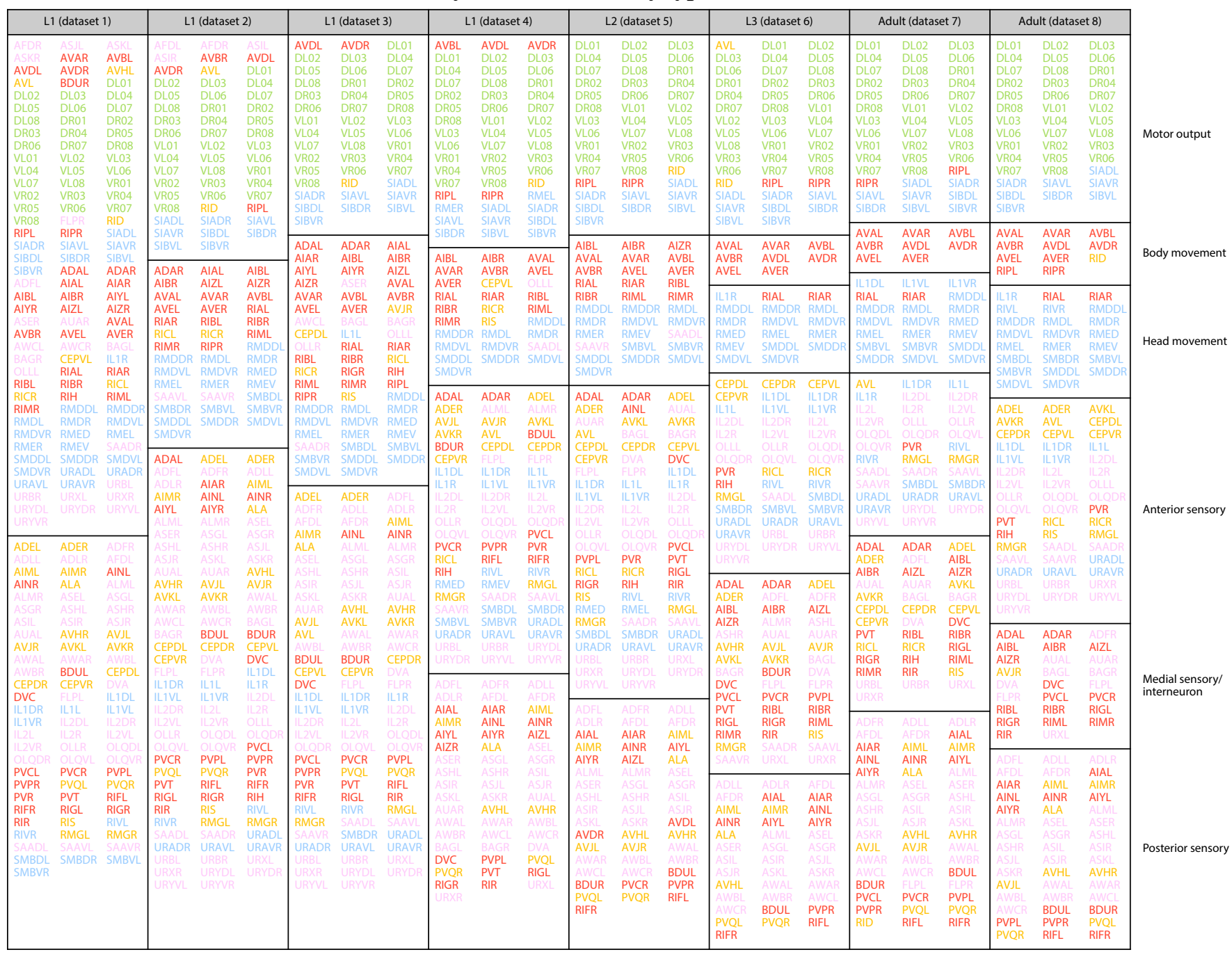


bioRxiv preprint doi: https://doi org/10.1101/2020.04 30.066209; this version posted May 22, 2020. The copyright holder for this preprint (which was not certified by peer review) is the author/funder, who has granted bioRxiv a license to display the preprint in perpetuity. It is made available under aCC-BY-NC-ND 4.0 International license.

Table S3. Optimal number of modules detected by WSBM using subsets of connections.

\begin{tabular}{|l|c|c|c|c|c|c|c|c|}
\hline $\begin{array}{l}\text { Connections } \\
\text { included }\end{array}$ & $\begin{array}{c}\text { L1 } \\
\text { (dataset 1) }\end{array}$ & $\begin{array}{c}\text { L1 } \\
\text { (dataset 2) }\end{array}$ & $\begin{array}{c}\text { L1 } \\
\text { (dataset 3) }\end{array}$ & $\begin{array}{c}\text { L1 } \\
\text { (dataset 4) }\end{array}$ & $\begin{array}{c}\text { L2 } \\
\text { (dataset 5) }\end{array}$ & $\begin{array}{c}\text { L3 } \\
\text { (dataset 6) }\end{array}$ & $\begin{array}{c}\text { Adult } \\
\text { (dataset 7) }\end{array}$ & $\begin{array}{c}\text { Adult } \\
\text { (dataset 8) }\end{array}$ \\
\hline $\begin{array}{l}\text { All } \\
\text { connections }\end{array}$ & 2 & 3 & 3 & 4 & 4 & 6 & 6 & 6 \\
\hline $\begin{array}{l}\text { Non-variable } \\
\text { connections }\end{array}$ & 2 & 2 & 2 & 2 & 2 & 4 & 5 & 5 \\
\hline $\begin{array}{l}\text { Stable } \\
\text { connections }\end{array}$ & 2 & 2 & 2 & 2 & 2 & 2 & 2 & 2 \\
\hline
\end{tabular}


bioRxiv preprint doi: https://doi.org/10.1101/2020.04 30.066209. this version posted May 22, 2020. The copyright holder for this preprin (which was not certified by peer review) is the author/funder, who has granted bioRxiv a license to display the preprint in perpetuity. It is made available under aCC-BY-NC-ND 4.0 International license.
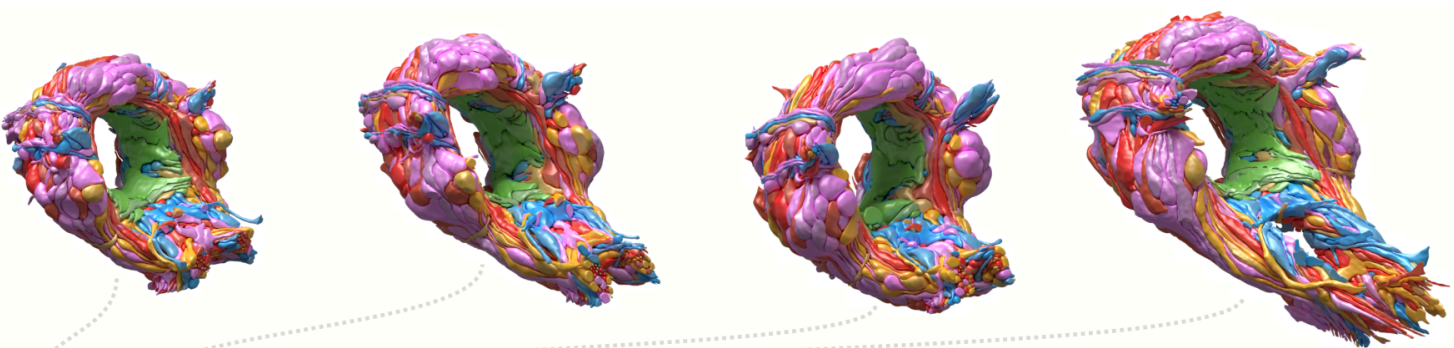

Neurons

Sensory

Modulatory

Inter

Motor

Muscle

Anterior
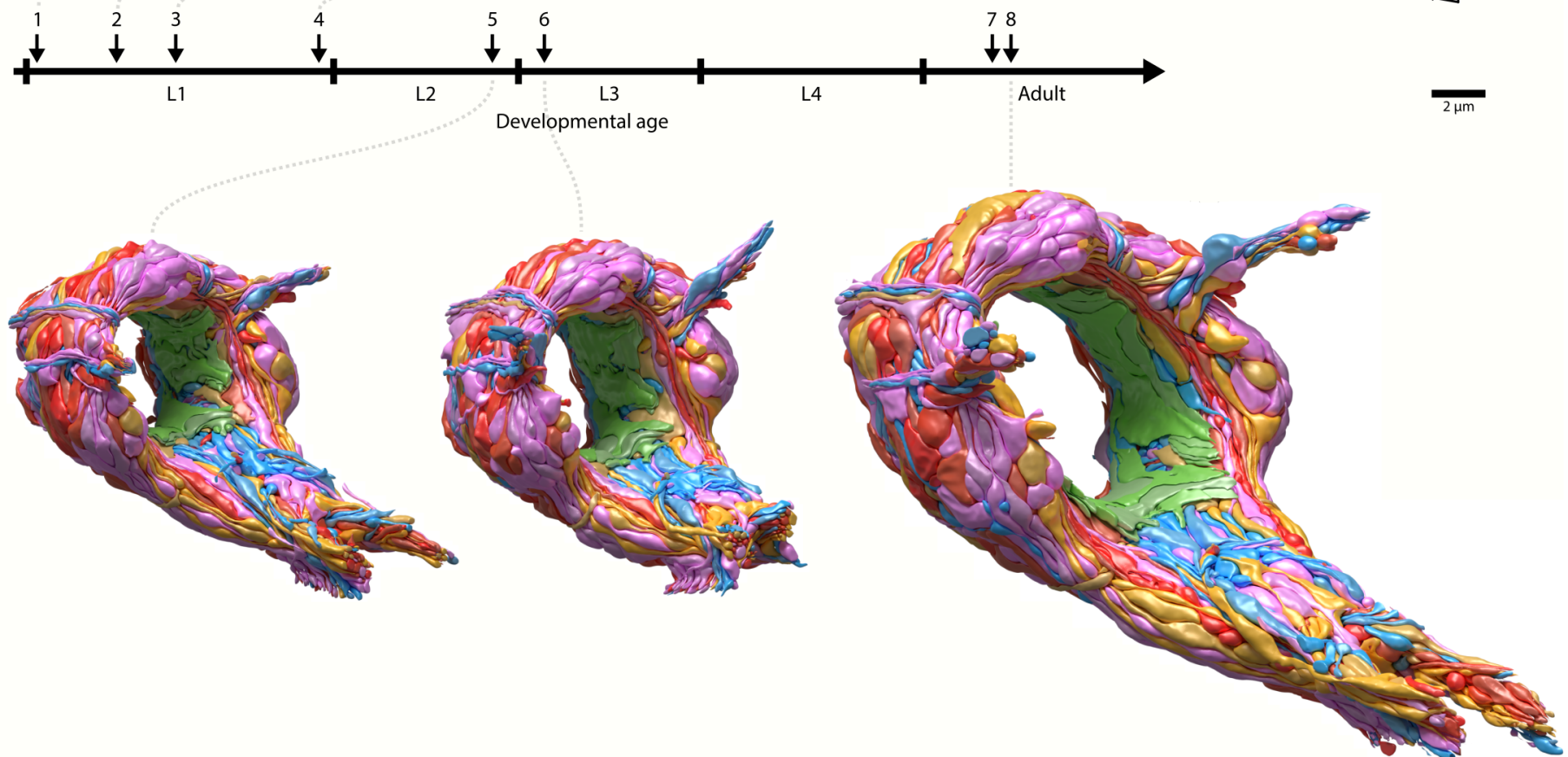

Figure S1. Volumetric models for seven $C$. elegans brains at respective developmental stages. All models include the complete neuropil of the brain, consisting of the nerve ring and ventral ganglion. Cells are colored by type. 
bioRxiv preprint doi: https://doi. org/10.1101/2020.04 30.066209; this version posted May 22, 2020. The copyright holder for this preprint (which was not certified by peer review) is the author/funder, who has granted bioRxiv a license to display the preprint in perpetuity. It is made available under aCC-BY-NC-ND 4.0 International license.

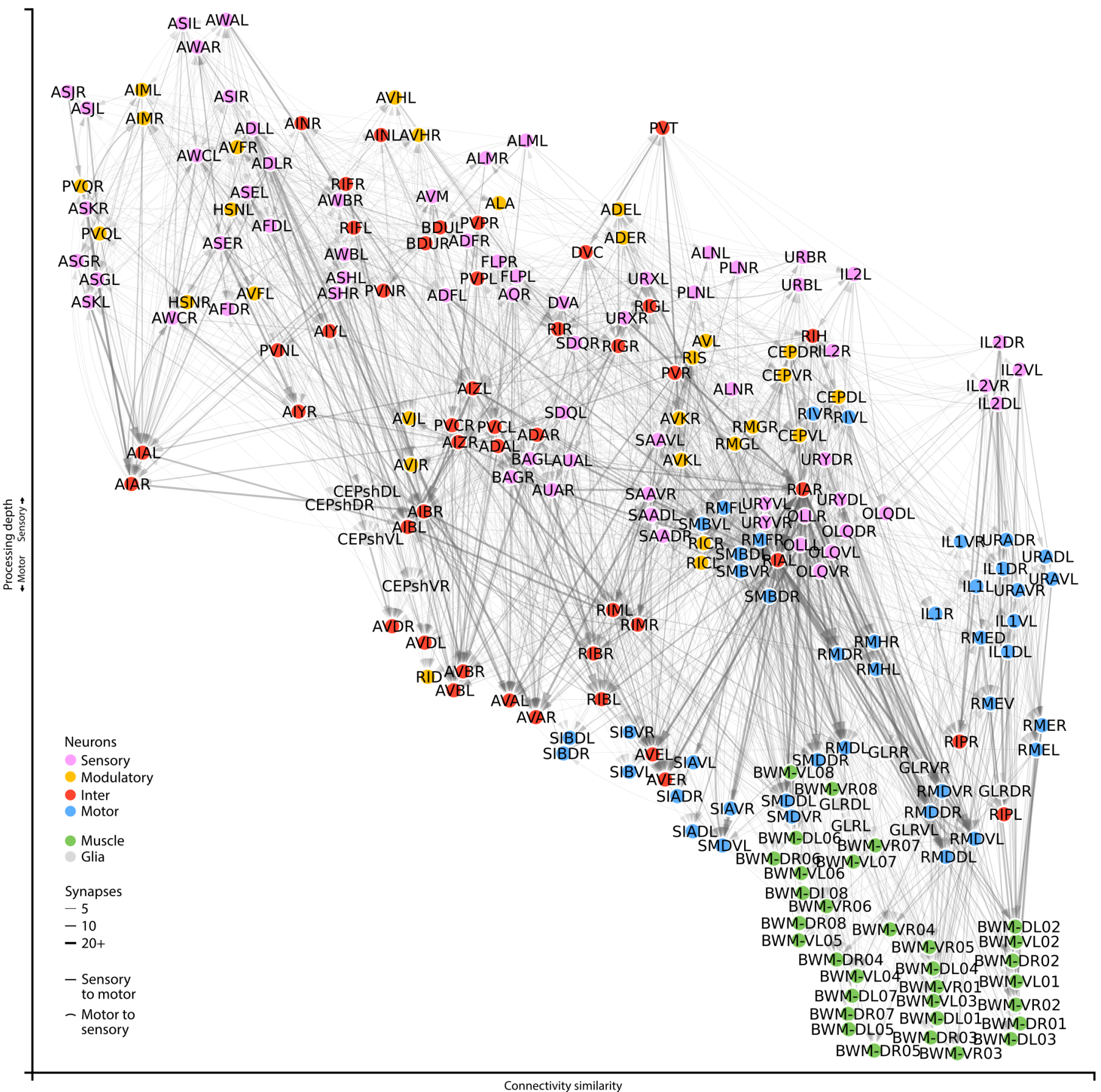

Figure S2. Closeup of an adult brain connectome. Wiring diagrams for an adult connectome (dataset 8). Each circle represents a cell. Circle colour denotes cell type. Each line represents a connection with at least one chemical synapse between two cells. Line width indicates synapse number. Straight lines direct information from sensory to muscle layers whereas curved lines direct information in reverse. Cell coordinates are represented as in Fig. 1b, with overlapping cells manually separated. 
bioRxiv preprint doi: https://doi.org/10.1101/2020.04.30.066209; this version posted May 22, 2020. The copyright holder for this preprint (which was not certified by peer review) is the author/funder, who has granted bioRxiv a license to display the preprint in perpetuity. It is made available under aCC-BY-NC-ND 4.0 International license.

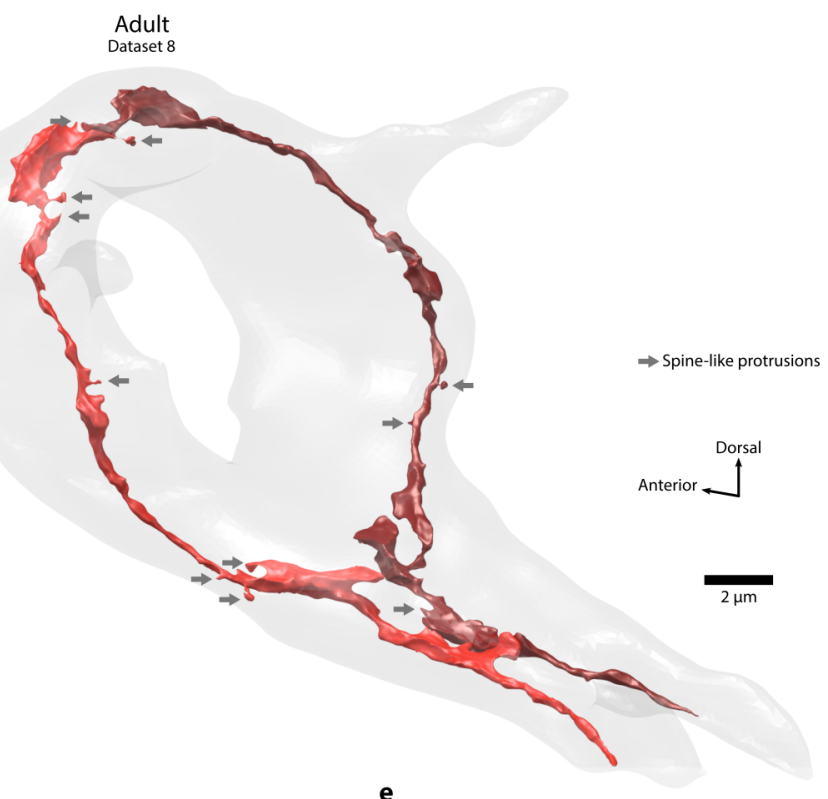

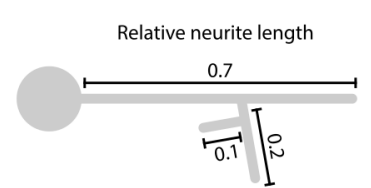

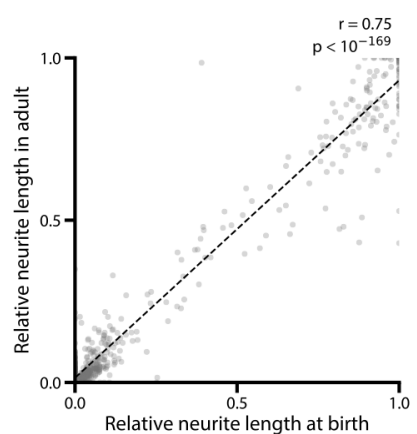

L3
Dataset 6
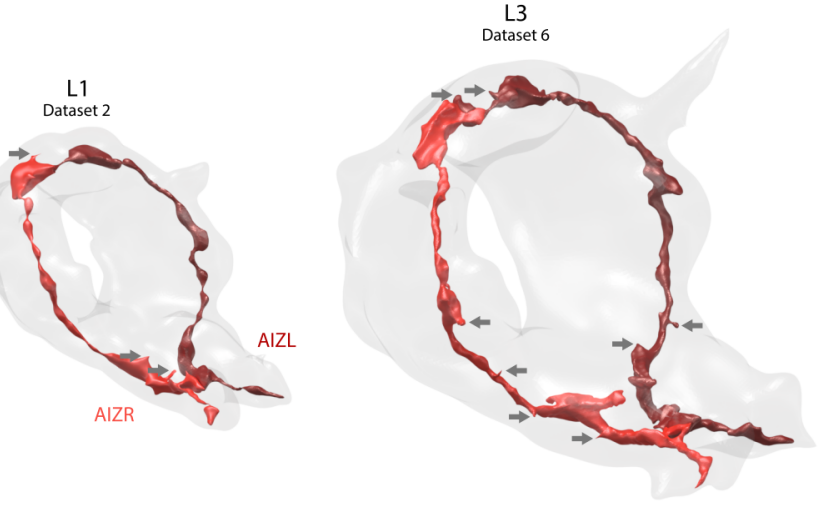

c
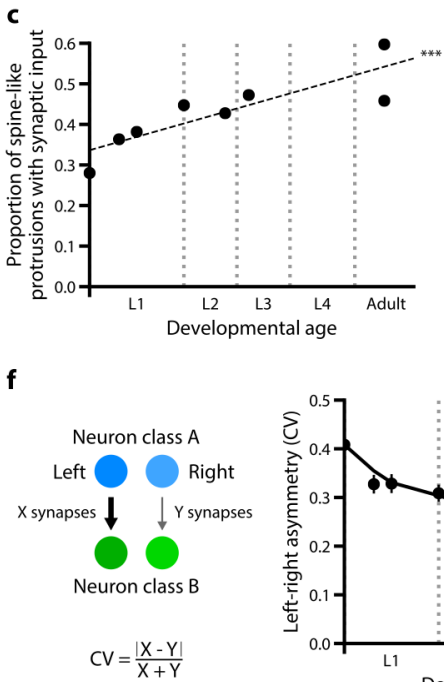

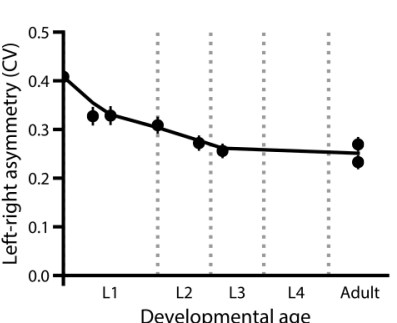

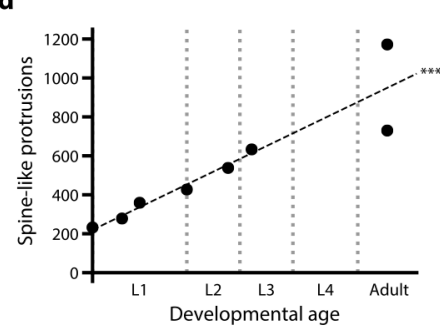

g
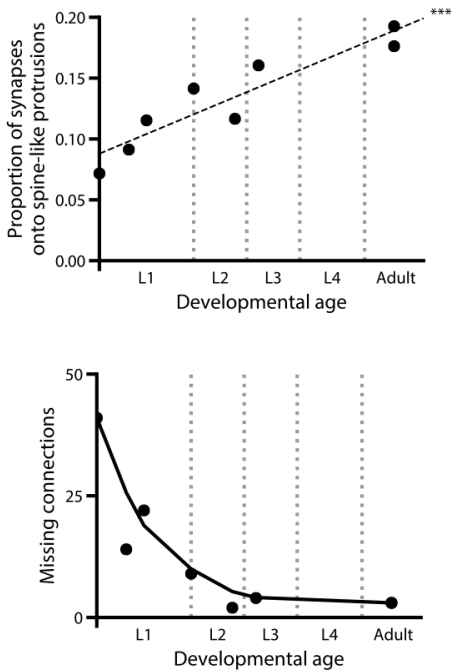

Figure S3. Neurites grow while maintaining overall brain geometry. a. 3D reconstructions of one neuron class (AIZL and AIZR) across maturation. The overall geometry was maintained, whereas the number of spine-like protrusions (grey arrows) increased over time. b. Correlation of the relative neurite length of each branch between $L 1$ (dataset 1 ) and adult (dataset 8). The length of each neurite is normalized against the total neurite length of the neuron. $p=1.7 \times 10^{-170}, r=0.75, n=949$, Spearman's rank correlation. $c$. Proportion of postsynaptic spine-like protrusions increases with maturation. ${ }^{* \star *} p=5.3 \times 10^{-7}$, Spearman's rank correlation. $d$. Total number of spine-like protrusions in the brain increases almost 5 -fold with maturation. ${ }^{* * *} \mathrm{p}=1.8 \times 10^{-4}$, Spearman's rank correlation. e. Proportion of synapses that have at least one spine-like protrusion postsynaptically increases with maturation. ${ }^{* * *} p=6.5 \times 10^{-5}$, Spearman's rank correlation. f. Connectivity asymmetry decreases from birth to adulthood, most significantly during L1. Asymmetry is defined as the coefficient of variation (CV) in synapse number between left-right cell pairs. Error bars indicate SE. g. Total number of missing connections decreases from birth to adulthood, most significantly during L1. A missing connection is defined as a connection absent in only one dataset and from one side of the brain. 
bioRxiv preprint doi: https://doi.org/10.1101/2020.04.30.066209; this version posted May 22, 2020. The copyright holder for this preprint (which was not certified by peer review) is the author/funder, who has granted bioRxiv a license to display the preprint in perpetuity. It is made available under aCC-BY-NC-ND 4.0 International license.

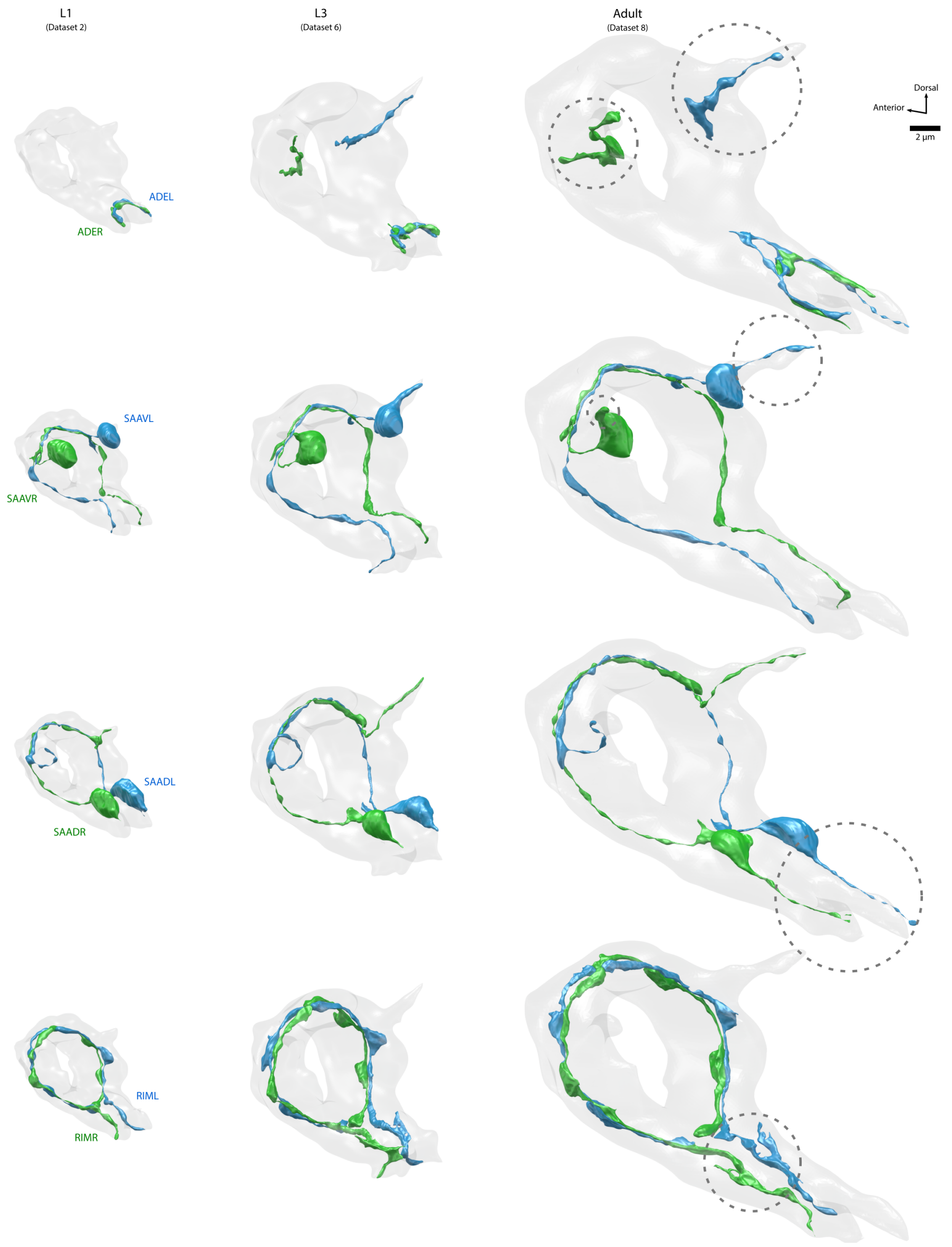

Figure S4. Three neuron classes grow new neurites after birth. Volumetric models of ADE, SAAV, SAAD, and RIM in L1 (dataset 2), L3 (dataset 6), and adult (dataset 8). These neurons pairs grow new major branches, highlighted by dotted gray circles. The new branches of ADE sprout outside the nerve ring. 
bioRxiv preprint doi: https://doi.org/10.1101/2020.04.30.066209; this version posted May 22, 2020. The copyright holder for this preprint (which was not certified by peer review) is the author/funder, who has granted bioRxiv a license to display the preprint in perpetuity. It is made available under aCC-BY-NC-ND 4.0 International license.

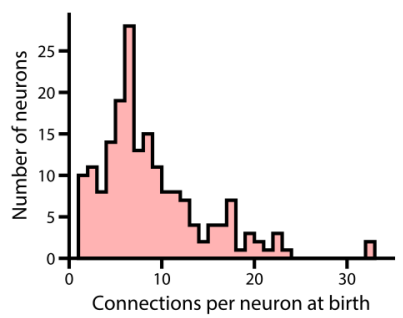

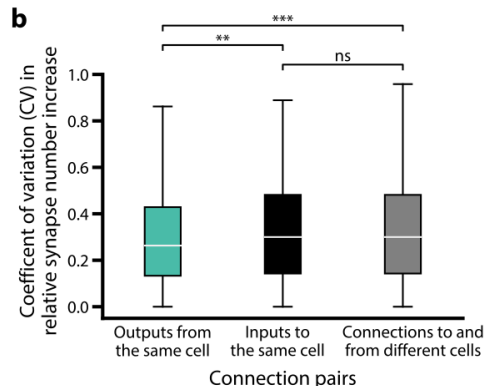

Figure S5. Non-uniform distribution of connections and strengthening of connections across maturation a. Distribution of the total number of input and output connections per neuron at birth. Some neurons have more connections than others.b. The relative number of synapses added to existing connections is correlated between outputs of the same cell compared to connections to and from different cells. The relative number of synapses added is quantified as the fold increase of synapse number from birth (dataset 1 ) to adulthood (averaged between datasets 7 and 8). ns (not significant) $p=0.30,{ }^{* *} p=2.8 \times 10^{-3},{ }^{* * *} p=6.2 \times 10^{-5}$, Mann-Whitney U test, FDR adjusted using Benjamini-Hochberg correction $\left(n_{\text {outputs }}=753, n_{\text {inputs }}=1203, n_{\text {other }}=90709\right.$ ). Center line, median; box limits, upper and lower quartiles; whiskers, $1.5 x$ interquartile range; outliers not shown. 
bioRxiv preprint doi: https://doi.org/10.1101/2020.04.30.066209; this version posted May 22, 2020. The copyright holder for this preprint (which was not certified by peer review) is the author/funder, who has granted bioRxiv a license to display the preprint in perpetuity. It is made available under aCC-BY-NC-ND 4.0 International license.

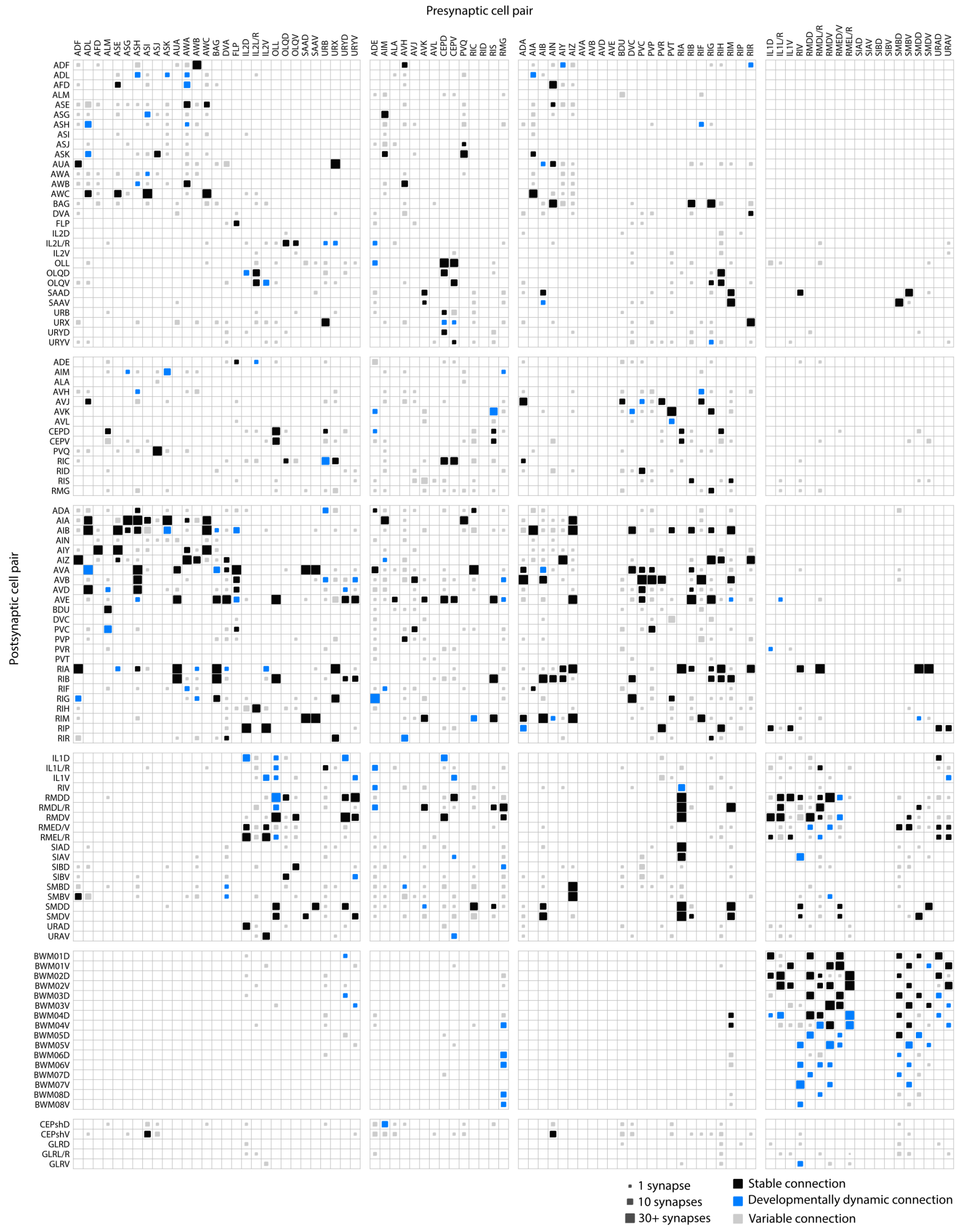

Figure S6. Connectivity matrix of the $\boldsymbol{C}$. elegans brain throughout maturation. A connectivity matrix that includes all connections observed in eight $C$. elegans brains. Cells are pooled by left-right pairs. The size of each connection represents its largest synapse number in any dataset. Stable, developmentally dynamic, and variable connections are colour-coded by their classification (see Methods). 
bioRxiv preprint doi: https://doi.org/10.1101/2020.04.30.066209; this version posted May 22, 2020. The copyright holder for this preprint (which was not certified by peer review) is the author/funder, who has granted bioRxiv a license to display the preprint in perpetuity. It is made available under aCC-BY-NC-ND 4.0 International license.

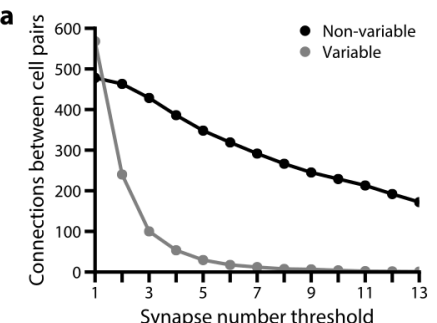

b

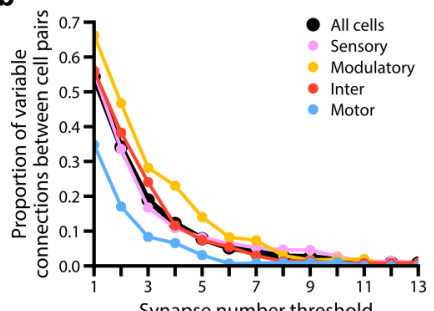

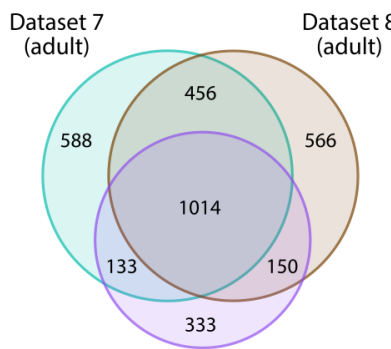

N2U, White et al., 1986

(adult)

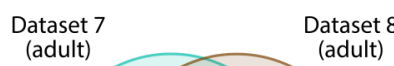

(adult)

variable connections

Adult-connectome defined non-variable connections
335

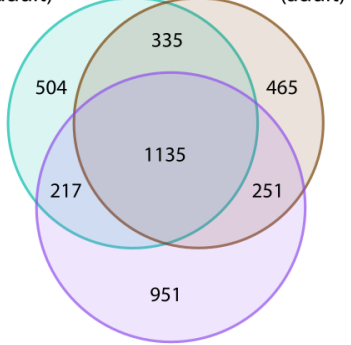

N2U, Cook et al., 2019 (adult)

Figure S7. Variable connections are prominent, even between animals of similar ages. a. Total number of both variable connections and non-variable (stable and developmentally dynamic) connections upon filtering by different synapse numbers in adults (averaged between datasets 7 and 8 ). No synapse number threshold provides a filter for specific removal of variable connections. Any threshold removes both variable and stable connections. b. Thresholding connections by synapse number leaves substantial proportion of variable connections for all cell types. The non-uniform distribution of variable connections is consistent when weak connections are excluded. c-d. The number of connections that are shared among or unique to the connectomes of our two adult datasets and the original adult connectome (c) annotated by White et al. ${ }^{25}$ and (d) reannotated by Cook et al. ${ }^{22}$ are illustrated in Venn diagrams (see Methods). We noted that most of the connections added in the reannotation by Cook et al. are not shared between the isogenic adult datasets described in this paper. Only $16 \%$ of the 1109 added connections are found in both our adult datasets. 
bioRxiv preprint doi: https://doi.org/10.1101/2020.04.30.066209; this version posted May 22, 2020. The copyright holder for this preprint (which was not certified by peer review) is the author/funder, who has granted bioRxiv a license to display the preprint in perpetuity. It is made available under aCC-BY-NC-ND 4.0 International license.
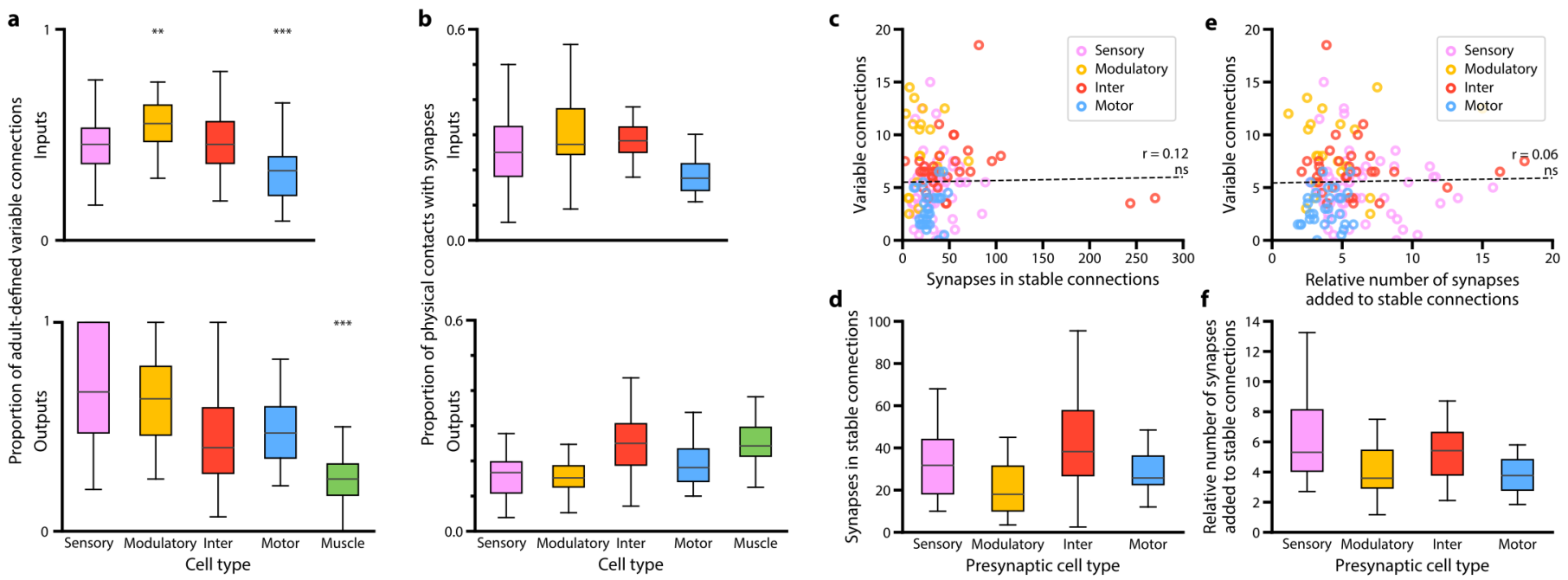

Figure S8. Propensity of forming variable connections correlates with cell type. a. Comparison of the proportion of adult connectome-defined variable and non-variable connections for each cell type. Adult-defined non-variable connections include the connections that are present in both of our adult datasets as well as the original connectome annotated by White et al. ${ }^{25}$ (see Fig. S7c). Cell types with significantly higher or lower proportions of variable connections are indicated, ${ }^{\star *} \mathrm{p}<10^{-2},{ }^{\star * \star} \mathrm{p}<10^{-3}, \mathrm{n}=28-65$, Mann-Whitney U test, FDR adjusted using Benjamini-Hochberg correction. Center line, median; box limits, upper and lower quartiles; whiskers, $1.5 x$ interquartile range; outliers not shown. b. The low variability of connections from motor neurons to muscles cannot be simply explained by saturation of their physical contacts by synapses. Physical contacts are not saturated for connections for any cell type. Motor neurons, which have the lowest proportion of variable connections (Fig. 4b), are not restricted by few available potential synaptic partners. Center line, median; box limits, upper and lower quartiles; whiskers, 1.5x interquartile range; outliers not shown. c-f. Higher variability for certain cell types could also not be simply explained by a fixed probability of an erroneous connection by neurons that exhibit abundant synapse formation. c. The number of variable connections formed by a cell does not correlate with the strength of its stable output connections. Each data point represents one cell. ns (not significant) $p=0.18, r=0.12, n=138$, Spearman's rank correlation coefficient. d. The number of synapses for stable output connections by cell types. Modulatory neurons, which exhibit a higher proportion of variable connections than other cell types (Fig. 4b), do not exhibit more synapses per stable connection. Center line, median; box limits, upper and lower quartiles; whiskers, 1.5x interquartile range; outliers not shown. e. The number of variable connections formed by a cell does not correlate with the number of synapses added to existing stable output connections from birth to adulthood. The relative number of synapses added is quantified as the fold increase of synapse number from birth (dataset 1 ) to adulthood (averaged between datasets 7 and 8). Each data point represents one cell. ns (not significant) $p=0.51, r=0.06, n=138$, Spearman's rank correlation coefficient. f. The relative number of synapses added to existing stable output connections by cell types. Connections from modulatory neurons, which have a higher proportion of variable connections than other cell types (Fig. 4b), do not exhibit higher increase in synapse number than connections from other cell types. Center line, median; box limits, upper and lower quartiles; whiskers, $1.5 \mathrm{x}$ interquartile range; outliers not shown. For panels b-f, the synapse number for the adult brain (averaged between datasets 7 and 8 ) is shown. 
bioRxiv preprint doi: https://doi.org/10.1101/2020.04.30.066209; this version posted May 22, 2020. The copyright holder for this preprint (which was not certified by peer review) is the author/funder, who has granted bioRxiv a license to display the preprint in perpetuity. It is made available under aCC-BY-NC-ND 4.0 International license.
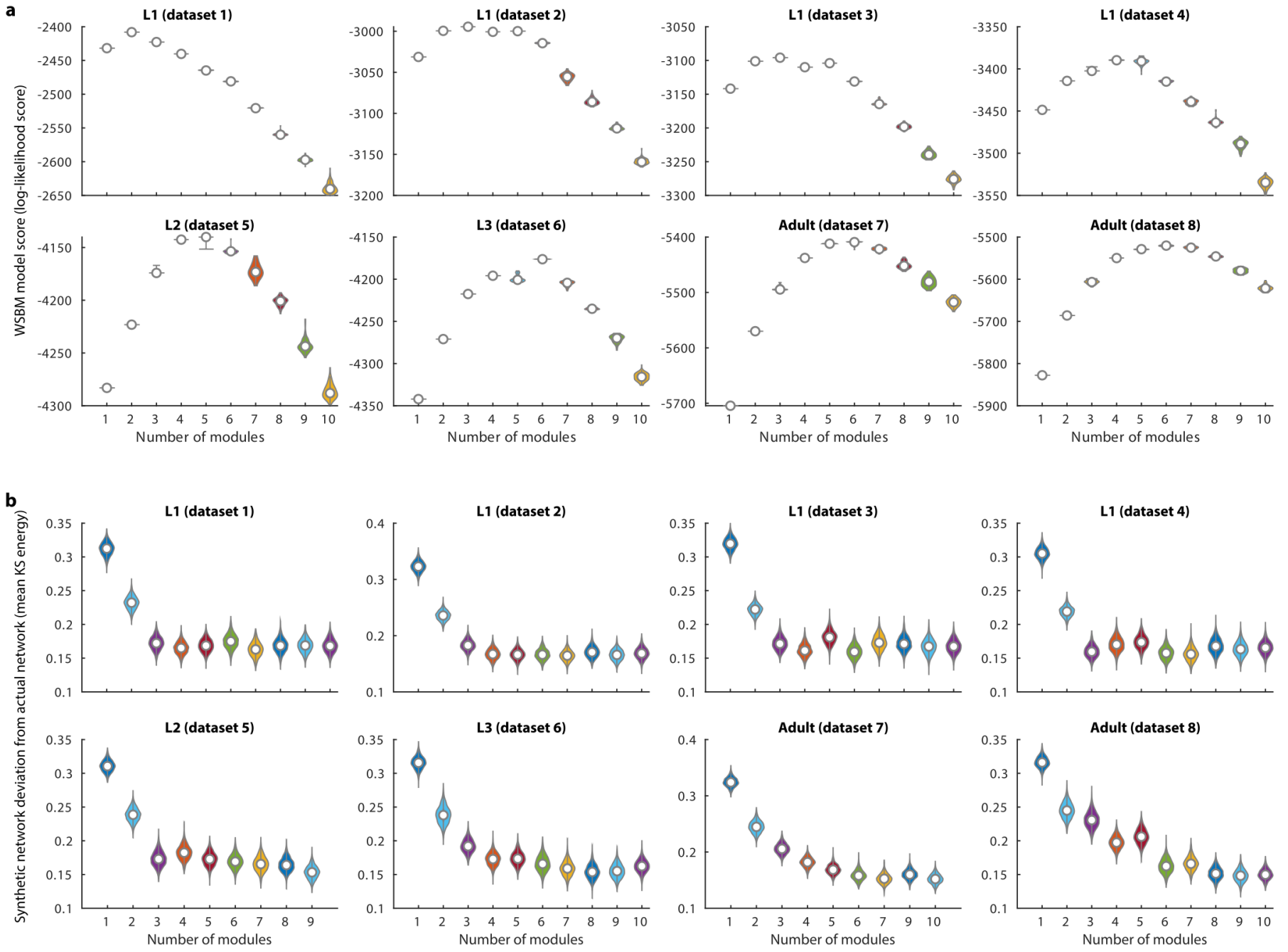

Figure S9. Cell modules across maturation. a. The log-likelihood score for each WSBM model (see Methods). b. The deviation between the connectome and each synthetic network generated from the best WSBM model, measured by the mean KS energy (see Methods). A lower deviation indicates a better match between the actual connectome and network generated from the model. Adult datasets show a clear preference to more than 5 modules, while juvenile datasets do not. 
bioRxiv preprint doi: https://doi.org/10.1101/2020.04.30.066209; this version posted May 22, 2020. The copyright holder for this preprint (which was not certified by peer review) is the author/funder, who has granted bioRxiv a license to display the preprint in perpetuity. It is made available under aCC-BY-NC-ND 4.0 International license.

\section{Supplemental text}

\section{Estimation of the developmental age of datasets}

The developmental age of each sample was established based on the described temporal cell division pattern exhibited by wild-type (N2) larva raised at $25^{\circ} \mathrm{C}^{30}$. Dataset 1: $\mathrm{L} 1$ at birth. No Q cell division, which occurs $\sim 3$ hours post-hatching (hrs) after birth, and Q cell nuclei are symmetrical, before nuclei migration, which occurs $\sim 2$ hrs after birth, placing this sample so close to birth, at $\sim 0$ hrs. Dataset 2: L1 at 5 hrs. The lack of H1 division, which occurs at $7.5 \mathrm{hrs}$ after birth, and no growth of PVC and SAA posterior neurites placed this sample to be $\sim 5$ hrs after birth. Dataset 3: L1 at 8 hrs. With $\mathrm{H} 1$ just completing its division and P5/6 starting their migration, this sample was placed at $\sim 8$ hrs after birth, when both events take place. Dataset 4 : L1 at the very end of the larval stage (16hr). This sample was estimated to be 16hrs, near the end of the L1 lethargus. It has two layers of cuticle. Both P11.aaa and P12.aaa have divided. V5R.p is in the midst of division, and H1.a has not yet divided; all happen at $~ 16$ hrs. Dataset 5: L2 towards the end of the larval stage (23hr). SML/R have not divided, which occurs at $\sim 29 \mathrm{hrs}$. It has 40 gonad cells, and a slight double cuticle that indicates the end of L2. However, its gut lumen contains food, placing this sample shortly before entering L2 lethargus, which occurs at $\sim 23 \mathrm{hrs}$. Dataset 6: L3 at $27 \mathrm{hrs}$. Based on the partial outgrowth of the RMF neurites, which is born at $23 \mathrm{hrs}$, this sample was estimated to be $\sim 27 \mathrm{hrs}$. Datasets 7 and 8: Young adults at $45 \mathrm{hr}$. Both samples have adult cuticles but are relatively small compared to other adults. The exact ages of the two young adult samples are uncertain, so they are treated as equals for analyses.

\section{Anatomical inconsistencies between samples}

A few major anatomic inconsistencies are observed in some datasets, likely due to heterogeneity or imprecision of development processes. These events do not have an impact on overall connectivity, as all non-variable connections between individual neuron classes were conserved. Dataset 2: CEPDL cell body is shifted to the anterior ganglion. Dataset 3: RIFL neurite terminates prematurely laterally, not reaching the dorsal midline. Dataset 4: RIH cell body is shifted to the anterior ganglion. PVCL and PVCR neurites both go right-handedly around the nerve ring, appearing as PVCR. Dataset 5: RMHL and RMHR neurites both transverse right-handedly around the nerve ring, appearing as RMHR. ADAL terminates prematurely at a dorsal sub-lateral position, not reaching the dorsal midline. Dataset 6: PVR neurite is fragmented. Dataset 7: RIFL and RIFR neurites both transverse righthandedly around the nerve ring, appearing as RIFR. 